\title{
European Journal of Physics
}

\author{
A Journal of the European Physical Society \\ published by \\ The Institute of Physics
}

Eur. J. Phys. was created to provide a regular channel of communication between physicists with responsibilities for teaching at the University level. It is concerned with techniques and ideas, with the sharing of experience and the improvement of teaching methods. It is also concerned with concepts and with problems - indeed with all subjects in physics that preoccupy those who have to initiate the dialogue with the next generation of physicists.

European in structure and in outlook, the Editorial Board under the Editor, Professor G.W. Series of Reading University, groups 10 members nominated by the European Physical Society and six by The Institute of Physics. Three numbers have already appeared and the last in the first volume will be out shortly.

\section{Scholarships}

Students and their department heads are reminded that EPS Scholarships are available in the countries listed below.

\section{Federal Republic of Germany}

Research Centres of DESY, Jülich, Karlsruhe, Hahn-Meitner Institute, GSI Darmstadt.

Italy

INFN Sections at the Universities of Bari, Bologna, Catania, Florence, Genoa, Milan, Naples, Padua, Pavia, Pisa, Rome, Trieste and Turin; also the ISS Rome and the national labs. of Frascati and Legnaro.

\section{The Netherlands}

\section{Institutes of FOM.}

\section{Poland}

Universities of Cracow, Gdansk, Gliwice, Otwock-Swierk, Warsaw, Wroclaw.

\section{Romania}

Seven different Institutes in Bucharest.

\section{Switzerland}

University of Geneva.

Also, EPS is collaborating with Ireland

Universities of Cork, Dublin and Galway. The Netherlands

University of Nijmegen.
If your institution is not a subscriber, sample copies may be obtained from:

The Marketing Dept. (Room 184)

The Institute of Physics

Techno House, Redcliffe Way

Bristol BS1 6NX, England

Subscription prices for libraries are:

$\begin{array}{cc}\text { Volume } 1 & £ 25 \\ \text { N. America } & \$ 49 \\ \text { Volume 2 } & £ 30\end{array}$

N. America $\$ 75$

Prices to members of EPS and its National Societies for personal use:

$\begin{array}{cc}\text { Volume } 1 & £ 12.50 \\ \text { Via EPS Secretariat } & \text { SwFr. 40 } \\ \text { Volume 2 } & £ 15 \\ \text { Via EPS Secretariat } & \text { SwFr. } 55\end{array}$

Via EPS Secretariat SwFr. 55

Members of EPS are invited to contribute to the journal as both authors and subscribers.

\section{Guide to Entries}

Europhysics News publishes annually three Meetings Issues: in March, June and November. The June issue contains also a directory section on the composition of the European Physical Society. In the March issue the information received to date is given in full; in the two subsequent issues some entries may be abbreviated and marked by an asterisk $\left({ }^{*}\right)$ indicating that full information can be found in the preceding Meetings issue of Europhysics News.

The present issue contains details of future events about which notification was received at the EPS Secretariat in Geneva by February 1981. They are listed in date order in two categories - Conferences and Schools. Europhysics Conferences, i.e. meetings organized by EPS, its Divisions and Sections are printed in bold type and boxed; events sponsored by EPS are printed in bold type; Europhysics Study Conferences are marked by an $\Theta$ preceding the date.

For inclusion in the June 1981 issue, organizers should send available details to the EPS Secretariat by 18 May.

\section{Istanbul Registrations}

Although no formal dead-line has been fixed for receiving registrations for the EPS 5th General Conference at Istanbul, 7-11 September, intending participants are advised to make their reservations early. EPS is in competition for hotel accommodation with all the other attractions of the Turkish resort. Block bookings have, of course, been made in advance for the Conference but these are rapidly filling up. Registration forms are available at EPS organized meetings and at the EPS Secretariat in Geneva.

\section{July Issue}

The July issue of Europhysics News will be a special issue devoted to lasers in which a review of the development of modern laser technology will be supported by articles on current and expected applications in various fields of science and engineering.

Organizers of conferences that are European in concept may apply for EPS sponsorship through the Secretariat.

\section{Key to Abbreviations}

A: Application deadline

$A b$ : Abstracts deadline

inv.: attendance restricted to invited applicants

NP: no proceedings will be published

$P$ : Papers deadline (information on proceedings has not been received)

PP: proceedings will be published.

The information is given in the following order:

Date

Venue

Title

Contact for information

A: .... / Ab: .... / PP, NP or P: Papers deadline / Number of participants / inv. / languages other than English / Sponsorship / Conference fee.

4 after the date indicates that there has been an important amendment to a previously published entry. 
For Guide to Entries see Front Page

\section{Conferences 1981}

\section{May} High-Energy Physics Experiments

Mrs. T. Jones, Microprocessor Conf. Secr. EF Division, CERN, CH-1211 Geneva 23 Ab: $31.12 .80 / 300$

\section{4-6 May}

Honolulu, Hawai, USA

Gradient-Index Optical Imaging Systems

Optical Society of America, Gradient-Index Meeting 1816 Jefferson Place, N.W., Washington, DC 20036 $A b: 16.1 .81 / P: 16.1 .81 /$ US $\$ 95$.

6-7 May

Enschede, NL

Dynamics Days Twente

R.H.G. Helleman, Theor. Physics, Twente Univ. of Technology, РОВ 217. NL 7500 AE Enschede

6-8 May

Noordwijkerhout, NL

Drie Landen Conferentie (Associations for Crystal Growth in NL, FRG, UK)

B Knook, Kamerlingh Onnes Laboratory, Nieuwsteeg 18, NL-2311 SB Leiden

6-8 May The Hague, NL

4th Int. Conference on Electrostatics

Electostatics $1981 \mathrm{c} /$ o KIvI, POB 30424 .

NL-2500 GK The Hague

Ab: $1.9 .80 / \mathrm{P}: 1.1 .81 / \mathrm{hFL} .520$ 125. - (students)

11-13 May

Berlin (West)

Annual Meeting: German Society of Rheology

K. Kirschke, German Soc. of Rheology, Unter den Eichen 87, D-1000 Berlin 45

A: 30.4.81/Ab: 30.11 .80 / NP: May $81 / 200$ / Engl. German / DM 150, - approx.

12-14 May

Paris, France

Journées de Chimie Physique - Surfaces et Interfaces: Changements de Phase et Transformation

C. Troyanowsky, Soc. de Chimie Physique, 10, rue Vauquelin, F-75231 Paris Cedex 05

Ab: $10.2 .81 / 200 /$ French / FF 300.- ;

75. - (students)

12-15 May

Grenoble, France

International Magnetics Conference (INTERMAG)

D. Randet, CEN, B.P. No 85 , Centre de Tri, F-38041 Grenoble Cedex

Ab: $15.12 .80 /$ PP: $23.3 .80 / 900 /$ US $\$ 165$.

12-15 May

Königstein, FfM, FRG

5th European Experimental NMR Conference

5th EENC, H. Caspari, I.N. Stranski Inst. Tech. Univ. Berlin, Strasse des 17. Juni 112, D-1000 Berlin 12

\section{8-20 May}

Santa Fé, NM, USA

International Conference on Plasma Science

S.J. Gitomer, Los Alamos Scientific Laboratory, Los Alamos, NM 87545

18-22 May

Flagstaff, AZ, USA

IAU Colloquium N $N^{\circ}$ 62: Current Techniques in Double and Multiple Star Research

R.S. Harrington, Naval Observatory, Washington, DC 20390

A: 15.2.81 / Ab: $15.4 .81 / \mathrm{PP}: 1.7 .81 / 50 /$ inv. / Engl., French / US $\$ 30$. -

18-22 May Sta. Barbara, CA, USA Workshop: Interaction of Particle Physics and Astrophysics

D.N. Schramm, Univ. of Chicago, Astronomy and Astrophys. Center, Chicago, IL. 60637

18-23 May

Riga, USSR

Int. Conf, on Defects in Insulating Crystals

V.M. Tuchkevich, Physics Inst.

Latvian SSR Academy of Ściences, IUPAP Salaspils - 1, SU-229021 Riga

19-21 May

1st European Conf. on Cineradiography with Photons or Particles

Mme Dionisi, Conf. Secretary, ANRT

101 ave. Raymond-Poincaré, F-75116 Paris

$A b: 1.10 .80 / P: 1.2 .81 /$ Engl., French
20-21 May

Molecules in Interstellar Space

London, UK

Executive Secretary. The Royal Society,

6 Cariton House Terrace, London SW1Y 5AG

20-23 May

Dubrovnik, YU

Forum on Universities in World Network of Information and Communication IV: Information Systems and Methods in Science and Technology

Ms. N. Tudor-Silovic, Referral Centre, Univ. of Zagreb POB 327, YU-41001 Zagreb

21-22 May

Risø, Denmark

Spring Meeting, Danish Physical Society

Atomic Physics: H. Knudsen

- Nuclear Physics: N. Rud

Institute of Physics, University of Aarhus, DK-8000 Aarhus C

A: $1.4 .81 / \mathrm{Ab}: 1.4 .81 / 100$

\section{2-27 May}

Grasse, France

IAU Colloguium No 63: High Precision Earth Rotation and Earth-Moon Dynamics; Lunar Distance and Related Observations

O. Calame, CERGA, Ave. N. Copernic, F-06130 Grasse

25-27 May

Baltimore, MD, USA

Johns Hopkins Workshop on Current Problems in

Particle Theory (5): Unified Field Theories and Beyond

K. Dietz, Physikalisches Institut, Universität Bonn, Nussallee 12, D-5300 Bonn

A: $15.4 .81 / 50 /$ inv

\begin{tabular}{|c|}
\hline $\begin{array}{l}\text { Poznan, Poland } \\
\text { Europhysics Study Conference: IRIS-1: } \\
\text { Internal Reorientation in Solids } \\
\text { Z. Pajak, Institute of Physics, } \\
\text { Grunwaldzka 6, PL-60780 Poznan } \\
70 \text { / inv. / US \$270.- (IOM), 300.- (others) }\end{array}$ \\
\hline $\begin{array}{l}\text { Hvar, Yugoslavia } \\
\text { Europhysics Study Conference on Dyna- } \\
\text { mics of Heavy lon Collisions } \\
\text { N. Cindro, Ruder Boskovic Institute, } \\
\text { Bijenicka 54, POB 1016, } \\
\text { YU-41001 Zagreb } \\
100 \text { / SFr. } 450 .- \text { (IOM); 500.- (others) all } \\
\text { incl. }\end{array}$ \\
\hline
\end{tabular}

\section{7-28 May}

Princeton, NJ, USA

Princeton University Plasma Physics Laboratory Information Meeting

E. Tanner, Plasma Physics Lab., Princeton Univ. Princeton, NJ 08544

27-28 May

Hajduszoboszlo, Hungar

Symposium on Light and Radiation Measurement

J. Schanda, Res. Inst. f. Technical Physics,

Hungarian Acad. of Sciences, Ujpest 1, POB 76 $\mathrm{H}-1325$ Budapest

28-31 May

Blacksburg, VA, USA

Physics in Collision: High Energy $\mathrm{e}^{+} \mathrm{e}^{-}$, ep, pp Interactions

P. Trower, Phys, Dept., Virginia Polytechnic and State Univ., Blacksburg, VA 2406

A: 15.3 .81 / PP / 200 / US $\$ 180 .-$ all incl.

1-4 June Munich, FRG

Laser 81 - Opto-Elektronik: 5 th Int. Congress and Trade Fair

Kongresszentrum, Laser 81 Opto-Elektronik, Postfach 1210 09, D-8000 Munich 12

1-4 June

Liblice Castle, CSSR

Mesons and Light Nuclei

E. Truhlik, Nuclear Physics Institute, CS $25068 \mathrm{Rez}$

A: $1.3 .81 / \mathrm{Ab}: 4.6 .81 / \mathrm{PP}: 4.6 .81 / 70 /$ inv. / DM 180.-

1-5 June

Cannes, France

$8^{\text {e }}$ Symposium International sur les Jets Moléculaires F.M. Devienne, Lab. de Physique Moléculaire des Hautes Energies, B.P. 2, F-06530 Peymeinade

1-5 June

8. Colloque sur le Traitement du Signal

Nice, France et de ses Application

Secrétariat du Colloque GRETSI, 7 chemin des Presses, B.P. 93, F-06802 Cagnes-sur-Mer

1-5 June
12th GIFT Int Seminar on Theoretical Physics: Non

Peñiscola, Spain Perturbative Aspects of Quantum Field Theory

J. Julve, Instituto de Estructura de la Materia, CSIC, Serrano 119, Madrid-6
1-7 June

Villars-sur-Ollon, Switzerland General Meeting on LEP (ECFA)

Ms. Ch. Redman, ISR, CERN, CH-1211 Geneva 23 A: $1.4 .81 / 300$

$4-5$ June

Leuven, Belgium

Assemblée Scientifique Générale de la Société Belge de Physique

P. Fettweis, Boeretang 200, B-2400 Mol

$\mathrm{Ab}: 27.3 .81$

45 June

Antwerp, Belgium

CECAM Workshop on Ab-Initio Calculation of Phonon Spectra

J. Devreese, U.I.A., Universiteitsplein 1, B-2610 Wilrijk

7-10 June

Roanoke, VA, USA

5th Rare Earth-Cobalt Magnet Workshop

K.J. Strnat, Univ, of Dayton, Dayton, $\mathrm{OH} 45469$

8-11 June

College Park, MD, USA

2nd Int. Conf. on Solid Films and Surfaces

R.L. Park, Dept. of Physics and Astrophys

Univ, of Maryland, College Park, MD 20742 $A b: 21.3 .81$

8-12 June

Gaithersburg, MD, USA

2nd int. Conf. on Precision Measurement and

Fundamental Constants

National Bureau of Standards, BIdg. 220, Room B 258 , Washington, DC 20234

A: 15.5 .81 / Ab: $2.2 .81 / \mathrm{PP}: 12.6 .81 / 250 /$ IUPAP CODATA / US $\$ 130 .-$ approx.

8 June - 1 Aug.

N. Hampshire/Calif., " USA

Gordon Research Conf: Frontiers of Science

8-12.6: Laser Diagnostics in Combustion (Chairman : J.W. Daily Vibrational Intensities (W. B. Person)

15-19.6: Nuclear Chemistry (A. Faessler)

Magnetic Resonance (M. Bloom)

Magnetic Resonance (M. Bloom)
Coatings and Films (H.S, Bender)

22-26.6.: Collisionless Shocks in Space, Astrophysical \& Labo ratory Plasmas IM. Walt)

- Physics of Steady - State Plasma Confinement (T.K. Chu) 29.6 - 3.7: Molecular Energy Transfer (Th. F. George)

Quantum Solids \& Fluids (A.M. Goldman, C.M. Varma)

6-10.7: Fiber Science IN.R.S. Hollies)

Liquid Crystals (D.W. Berreman

Physical Metallurgy (G.T. Hahn)

Chem. \& Phys. of Solids (W.F. Brinkman, T.H. Geballe) Atomic Physics IS.R. Lundeen)

Fuel Science (C.T. Ratcliffe

13-17.7: Thin Films \& Solid Surfaces (G. Haacke) Solid State Studies in Ceramics (L.C. De Jonghe) Line \& Point Defects in Semiconductors (G.D. Watkins

27-31.7: Thermodynamic Analysis (K.S. Spiegler) Dynamics of Molecular Collisions (R.J. Cross)
7.8: Flow in Permeable Media (R.L. Reed, R.S. Schechter) 7.8: Flow in Permeable Media (R.L. Reed, R.S
Nonlinear Optics and Lasers (D. Auston) Nonlinear Optics and
Glass (P.C. Schultz)

10-14.8: Remote Sensing of Earth's Surface from Space

(J. Estes)
Few.Body Problems in Chem. \& Phys. IR.G. Newton Chem. \& Phys. of Liquids (R.L. Scot) Dyclear Structure Phyics iP. Axell

s (M.J. Cardillo)

8: Science of Adhesion (M.L. Hair)

Elementary Particle Interactions (R. Thun

A.M. Cruickshank, Director, Gordon Research

Conferences, Pastore Chemical Laboratory

University of Rhode Island, Kingston, RI 02881

$100 /$ inv. / US $\$ 220 .-$ (280. - Calif.)

B-12 June
Europhysics Conference: Nuclear and Ato-
mic Physics with Heavy lons
I.A. Dorobantu, Central Institute of Phy-
sics, POB MG-6, Bucharest
A: 1.5 .81 / Ab: 15.4.81 / PP: 15.5.81 / 300/
English / SFr. 110. - (IOM); 140.-
(mbrs. Nat. Soc.); 180. - (others)

9-13 June

Bremen, FRG

Joint Conf.: German Soc. for Applied Optics and VDI/VDE Soc. for Fine Precision Techniques

H.-J. Preuss, c/o Ernst Leitz Wetzlar GmbH,

Postfach 2020, D-6330 Wetzlar

Ab: 30.1 .81 / DM 80.-; 10.- (students)

10-12 June

Washington, DC, USA

Conference on Lasers and Electro-Optics (CLEO 80)

J. Quinn, Optical Society of America,

1816 Jefferson Place, N.W. Washington, DC 20036

Ab: 12.1 .81 US $\$ 95 .-(20 .-$ full time students $)$

10-12 June

Ann Arbor, MI, USA

5 th Symposium on $X$ - and Gamma-Ray Sources and Applications

Ms. C. Lehmann, Phoenix Memorial Lab., University of Michigan, Ann Arbor, MI 48109 


\section{4-24 June}

Bechyne, CSSR

Selected Topics in Quantum Field Theory and Mathe matical Physics

J. Niederle, Inst. of Physics, CSAV, Na Slovance 2, CS-18040 Prague 8

A: 31.3.81 / PP / 120 / Engl. / US \$ 120.- approx

15-17 June

Int. Microwave Symposium

Los Angeles, CA, USA

A. Clavin, Hughes Aircraft Company, Bldg. 268

8433 Fallbrook Ave., Canoga Park, CA 91304

\section{5-17 June}

Tullahoma, TN, USA

Engineering Aspects of Magnetohydrodynamics

M.H. Scott, Energy Conversion Div., Univ, of

Tennessee Space Institute, Tullahoma, TN 37388

\section{5-18 June}

Halifax, N. Scotia, Canada

Annual Congress: Canadian Association of Physicists Dept. of Physics, Dalhousie Univ., Halifax, Nova Scotia

\section{5-18 June}

Rolla, MO, USA

15th Rare Earth Research Conference

W.J. James, Materials Research, University of Missouri. Rolla, MO 65401

15-19 June

Pasadena, CA, USA

IUTAM Symposium: Mechanics and Physics of Bubbles in Fluids

M.S. Plesset, Cal. Inst. of Technology, Engng. Science Dept., Pasadena, CA 91125

\section{5-19 June}

Madrid, Spain

Jornadas Ibericas - Optica 81 : Integrated Optics and Optical Fibres

O. Soares, Lab. Fisica, Fac. Ciencias. P. 4000 Porto, Portuga

A: $30.4 .81 /$ PP / $100 /$ English

\section{5-19 June}

Gaithersburg, MD, USA

8th Symposium on Thermophysical Properties

J.V. Sengers, Inst. for Phys. Science and Technology, Univ. of Maryland, College Park, MD 20742

15-20 June

Barcelona, Spain

Workshop: Relativistic Action at a Distance (Classical and Quantum Aspects)

X. Fustero, Dept. of Theor. Physics, Univ. of Barcelona, Diagonal 647, Barcelona 28

A: 14.4 .81

\section{7-19 June}

Dublin, Ireland

2nd Int. Conf, on Numerical Analysis of Semiconduc. tor Devices and Integrated Circuits

NASECODE II Conf., 39 Trinity College, Dublin 2 $A b: 20.3 .81$

21-26 June Notre Dame, IN, USA

12 th Int. Symposium on Multiparticle Dynamics

W. Shephard, Notre Dame University

Notre Dame, IN 46556

\section{2-23 June}

Palo Alto, CA, USA

5 th Computational Fluid Dynamics Conference

American Inst. of Aeronautics and Astronautics Inc. 1290 Avenue of the Americas, New York, NY 10019

\section{2-24 June}

Trondheim, Norway

Annual Meeting of the Norwegian Physical Society

$\mathrm{H}$. Olsen, Dept. of Physics, Univ. of Trondheim, NLHT, N-7055 Dragvoll

A: 15.5.81 / Ab: 15.5.81 / NP / Engl., Norw. nKr. 150.-

22-24 June

16th Thermophysics Conference

. 1290 Avenue of the Americas, New York. NY 10019

\section{2-25 June}

Berkeley, CA, USA

2nd Int. Topical Meeting: Photoacoustic Spectroscopy

Optical Soc. of America, Photoacoustic Spectroscopy, 1816 Jefferson PI., N.W., Washington, DC 20036 $A b: 6.3 .81 / P: 6.3 .81 /$ US $\$ 100$.-

\section{2-26 June} Amsterdam, NL

Int. Symposium on X-Ray Astronomy

R.D. Andresen, Space Science Dept. ESTEC, Postbus 299, NL-2200 AG Noordwijk Ab: $1.4 .81 / 150 / \mathrm{hFI}, 150$.

\section{3-25 June}

Palo Alto, CA, USA

14th Fluid and Plasma Dynamics Conference

American Inst. of Aeronautics and Astronautics Inc. 1290 Avenue of the Americas, New York, NY 10019

23-26 June

Dundee, UK

9 th Dundee Biennial Conf. on Numerical Analysis

G.A. Watson, Dept. of Mathematics,

Univ. of Dundee, Dundee DD14HN

\section{NUCLEAR \& ATOMIC PHYSICS WITH HEAVY IONS \\ Bucharest, 9-12 June, 1981}

The Conference will be held on the campus of the Faculty of Physics, close to the Central Institute of Physics and $13 \mathrm{~km}$ from Bucharest. The site offers a first class hotel, student homes, restaurant, cafeteria, etc. and a regular bus service to the city. Accommodation will be arranged mainly on the campus but it can also be provided in the city upon very early request.

Participants are kindly requested to observe the application deadline of 1 May (see entry opposite). No special application form is needed. Final organizational details will be sent to all participants together with the acceptance letter.
Invited speakers

R. BOCK (Darmstadt)

F. BOSCH (Darmstadt)

R. DIAMOND (Berkeley)

M. ERICSON (CERN)

W. GREINER (Frankfurt/Main)

M. IVASCU (Bucharest)

E. MERZBACHER

(Chappel Hill)

R. RICCI (Padua)

D.K. SCOTT (Michigan)

R. SIMON (Darmstadt)

I. TALMI (Rehovoth)

I. URSU (Bucharest)

P. von BRENTANO (Cologne)

J. WILCZYNSKI (Cracow)
A. FAESSLER (Tübingen)
Recent developments in experiments with heavy ions. Superheavy quasiatoms.

Continuum gamma ray spectroscopy.

Nuclear matter at high densities: pion condensation. High spin states in nuclei.

Q E D of strong fields.

Nuclear spectroscopy at $80 \leqq A \leqq 90$.

The interplay between nuclear and atomic phenomena in ion-atom collisions.

Experiments with light heavy ions.

Review of experimental results in heavy ion collisions at inter mediate energies.

Crystal ball gamma ray spectroscopy.

The interacting Boson model.

Electronic structure of the uranium ions in crystals.

Nuclear spectroscopy around the double closed shells $\mathrm{Z}=64, \mathrm{~N}=82$ Incomplete fusion reactions

\section{3-26 June} Implantation

R.P.M. Procter, Corrosion and Protection Centre, UMIST, POB 88, Manchester M60 10D $\mathrm{Ab}: 1.5 .81$

25-27 June Tokyo, Japan

INS Symposium on Quark and Lepton Physics

Ms. F. Nunokawa, INS, University of Tokyo, Tanashi, Tokyo 188

\section{25-27 June Erice, Italy \\ Europhysics Study Conf.: C
for Physicists in Europe \\ W.A. Lingeman, IKO, POB 4395 , NL-1009 AJ Amsterdam

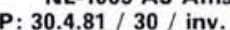

29 June - 1 July Bozeman, Montana, USA 41st Annual Physical Electronics Conference

G.J. Lapeyre, Physics Dept.

Montana State University, Bozeman, MT 59717

$A b: 1.5 .81$

29 June - 1 July

Colloguium on (in conj, with Gen. Congress of French Phys. Soc.)

P. Fauchois, Lab. de Thermodynamique.

UER des Sciences, Univ, de Limoges,

123 rue Albert Thomas, F-87060 Limoges Cedex

29 June - 3 July

Grand Unified Theories and Related Topics

Z. Maki, Research Inst. for Fundamental Physics, Kyoto University, Kyoto 606

29 June - 3 July

Grand Unified Theories and Related Topics

Z. Maki, Research inst, for Fundamental Physics Kyoto University, Kyoto 606

29 June - 3 July Palaiseau, France

4th Int. Topical Conf. on High-Power Electron and Ion-Beam Research and Technology

H. Doucet, Lab. de Physique des Milieux lonisés. Ecole Polytechnique, F-91128 Palaiseau

Ab: 1.3.81/English

29 June - 3 July
5 th Int. Conf. on Laser Spectroscop
Takeshi Oka, Herzberg Inst. of Astroph Jasper Park, Canada IUPAP National Research Council, Ottawa, Ont. K1A OR6

\section{June - 4 July}

Clermont-Ferrand, France Congrès Général de la Société Française de Physique J.L. Irigaray, UER, B.P. 45, F-63170 Clermont-Ferrand A: 15.3.81/Ab: 15.3.81/PP: $4.7 .81 / 600 /$ French, Engl. / FF 320. - (IOM \& mbrs SFP) 450. - (others); 150 - (junior mbrs SFP)
30 June-2 July Amsterdam, The Netherlands Int. Conf.: Ion and Plasma Assisted Techniques (IPAT 81

Secretariat, IPAT 81, 26 Albany Street, Edinburgh EH1 3QH, UK

Ab: 11.11 .80 / PP: 25.2 .81 / hFl. 390.- (IOM); 430. - (others); 85. - (students); incl. proc.

30 June - 2 July

Oxford, UK

7 th Thermodynamics and Fluid Mechanics Convention Conf. Dept., Institution of Mech. Engrs.

1 Birdcage Walk, London SW1H 9JJ

30 June - 3 July

Conf. on Differential Geometric Methods in Theoretical Physics

International Centre for Theoretical Physics, POB 586, 1-34100 Trieste

30 June - 3 July

Arco, L. Garda, I

P. Mazzoldi, REI 81, IRST, I-38050 Povo

A: $3.4 .81 / \mathrm{Ab}: 1.3 .81$

1-8 July

Wailea, Hawaii

Int Conf, on Neutrino Physics and Astrophysics (Neutrino-81)

V.z. Peterson, Dept. of Physics and Astronomy, University of Hawaii, 2505 Correa Rd, Honolulu, inv. Hawaii 96822 USA

2-8 July Grenoble, France 9 th Int. Conf. on Amorphous and Liquid Semiconductors

Conf. Secretariat, Groupe des Transitions de Phases, CNRS, 166X, F-38042 Grenoble Cedex

A: 15.5 .81 / Ab: 13.2.81/PP: 15.5.81/IUPAP / FF 750. - incl. proc.; 400. - Istudents, not incl. proc.

5-10 July Jerusalem, Israel

4th Int Conf, on Surface and Colloid Science A.S. Kertes, Inst. of Chemistry, The Hebrew University, Jerusalem 400 / IUPAC

6-9 July

Lausanne, Switzerland

7th Int. Conf. on Internal Friction and Ultrasonic Attenuation in Solids (ICIFUAS - 7)

W. Benoit, Laboratoire de Génie Atomique,

33 av. de Cour, $\mathrm{CH}-1007$ Lausanne

A: 16.4 .81 / Ab: 31.10 .80 / PP: $15.3 .81 / 300$ / SFr. 200.-

6-9 July

Strasbourg, France

27th IUPAC Int. Symposium on Macromolecules

Macro 1981, Société de Chimie Industrielle. IUPAC
Radiation Effects in Insulators 
6-10 July

Versailles, France

9th Int. Conf. on High Energy Physics and Nuclea Structure ( 9 - ICOHEPANS)

9 - ICOHEPANS, B.P. No. 2

F-91191 Gif-sur-Yvette Cedex

A: 31.3.81 / Ab: 6.4.81 / PP / 700 / Engl., French IUPAP / FF 500. - incl. proc.

6-10 July Lyon, France

9th Int. Conf. on Atomic Collisions in Solids

9th ICACS, Institut de Physique Nucléaire,

Université Claude Bernard, Lyon - I,

43 bd. du 11 Novembre 1918,

F-69622 Villeurbanne Cedex

A: 31.3.81 / Ab: 31.3.81 / PP / 350 / Engl., French / FF 650. - incl. proc.; 300. - (students)

6-10 July Trieste, Italy

Int. Conf. on Physics of Intercalation Compound

L. Pietronero, Brown Boveri Research Centre, CH-5405 Baden

A: May 81 / PP / 80 / inv. / SFr. 80. - (IOM) 100. - lothers)

7-10 July
IAU Coll.: Automated Data Retrieval in Astronomy

C. Jaschek, Observatoire, 11, rue de l'Université F-6700 Strasbourg

7-10 July

Mrance 3rd Int. Conf. on Hot Carriers in Semiconductor J-P. Nougier, Université des Sciences et Tech niques du Languedoc

Centre d'Etudes d'Electronique des Solides, F-34060 Montpellier Cedex

Ab: 1.3.81 / PP / 200 / FF 550. - incl. proc. 650. - after 1.6.81

8-10 July

Swansea, UK

8th Annual Conf. on Plasma Physics

C.N. Lashmore - Davies, Culham Lab.

Abingdon OX14 3DB

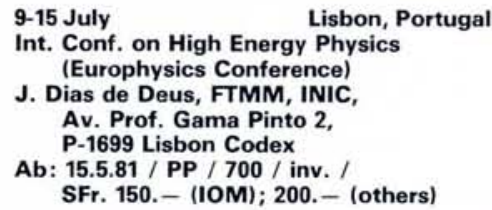

12-17 July

Exeter, UK

th International NMR Meeting

J.F. Gibson, The Royal Society of Chemisty Burlington House, London W1V OBN

Ab: $1.2 .81 /$ f 40 . - approx

13-17 July

Srinagar, India

Int Conf on the Applications of Mössbauer Effect

V. G. Bhide, Nat. Physical Laboratory, Hillside Road, New Delhi 110012

PP / IUPAC / US \$ 100 .

13-25 July

17th Int. Cosmic Ray Conference

Paris, France

T. Montmerle, CEN Saclay, DPh/EP/AP,

F-91191 Gif-sur-Yvette Cedex

Ab: 1.4.81 / PP: $1.4 .81 / 1000$ / iny. / Engl. / IUPAP / FF 600. - incl. proc.

14-16 July

Strasbourg, France

Recent Theoretical (Computational) Developments in Atomic Collisions in Solids

Y. Ohtsuki, Dept, of Physics, Waseda University,

Ohkubo 3, Shinjuku, Tokyo 160, Japan

14-18 July

Minsk, USSR

15th Int. Conf. on Phenomena in lonized Gases

Organizing Committee, ICPIG XV, Institute of Physics, BSSR Academy of Sciences, Leninskii Prospekt 70 Minsk 220602

$A b: 2.1 .81 /$ IUPAP

15-21 July

Gatlinburg, TN, USA

12th Int. Conf. on the Physics of Electronic and Atomic Collisions

S. Datz, Oak Ridge National Lab., POB X, Oak Ridge, TN 37830

$A b: 13.3 .81 /$ IUPAP

20-23 July

Prague, CSSR

22nd Prague Microsymposium on Macromolecules

PMM Secretariat, Heyrovského nam.

CS 16206 Praha 6-Petriny

A: $1.5 .81 / \mathrm{Ab}: 15.5 .81 / \mathrm{PP}: 1.7 .81 / 130 /$ IUPAC US $\$ 80 .-$ approx
20-24 July

1981 Int. Conf. on Luminescence

Berlin (West)

. Broser, Institut für Festkörperphysik II,

Technische Universität Berlin,

Strasse des 17. Juni, 112, D-1000 Berlin 12

Ab: 15.1 .81 / PP: 15.6 .81 / 800 / IUPAP /

DM 250.-; 50. - (students).

After 20.5.81: DM 300. - and 80.-

20-24 July

Warwick Univ., UK

4th Int. Conference: Software Engineering for Tele communication Switching Systems

EE Conference Department, Savoy Place. London WC2R OBL

21-24 July Seattle, WA, USA

Conf. on Nuclear and Space Radiation Effects

Arimura, Bœing Aerospace Co., 2R 00

POB 3999, Seattle, WA 98124

25-28 July

Berlin (West)

7 th Int. Conf. on Conduction and Breakdown in Dielectric Liquids

W.F. Schmidt, 7 ICDL, Bereich Strahlenchemie, Hahn-Meitner-Institut f. Kernforschung. D-1000 Berlin 39

29-31 July

Regensburg, FRG

dhe Excited States of Ions and Molecules in Solids (DPC - 81)

W. Gebhardt, Universität Regensburg,

Fakultät $f$. Physik, Universitätsstr. 31

D-8400 Regensburg

3-7 Aug

Albuquerque, NM, USA

AU Symposium No, 97: Extragalactic Radio Sources K.1. Kellerman, NRAO, POB 2

Green Bank, WV 24944

$\mid A U$, URSI

8-13 Aug.

Boston, MA, USA

23rd Annual Meeting of American Association of Physicists in Medicine

American Association of Physicists in Medicine,

III E. Wacker Drive, Chicago, IL 60601

10-12 Aug

Copenhagen, Denmark

Nordic Solid State Physics Conference

N.H. Andersen, Physics Dept., Risø National Lab.

DK-4000 Roskilde

A: $1.7 .81 / A b: 1.5 .81 / P P: 1.7 .81 / d K r .300 .-$

10-14 Aug.

San Diego, USA

Cryogenic Engineering Conference

Int. Cryogenic Materials Conference

Dee Belsher, National Bureau of Standards,

Boulder, CO 80303

$A b: 1.3 .81 / P P: 10.8 .81 / 500$

10-14 Aug

Boulder, CO, USA

Int. Conf, on Low - Dimensional Conductors

E.M. Conwell, Xerox Webster Research Center

800 Phillips Rd. W 114, Webster, NY 14580 US $\$ 100$.

10-14 Aug.

Bad Honnef, FRG

Int. Symposium on the Physics of Solids under High Pressure

J.S. Schilling, Experimentalphysik IV Universität Bochum, D-463 Bochum 1

A: 1.4.81/PP: $10.8 .81 / 70 /$ inv.

10-14 Aug. Edinburgh, UK

th Int. Symposium on Plasma Chemistry

B. Waldie, Dept. of Chem, and Process Engng.

Heriot-Watt University, Chambers St.

Edinburgh $\mathrm{EH} 11 \mathrm{HX}$

$A b:$ Jan. 81 / PP: May $81 /$ IUPAC

11-20 Aug.

Berlin (West)

6th Int. Conf. on Mathematical Physics - Congress of Association for Mathematical Physics

R. Seiler, Institut $f$, theor. Physik, FU Berlin, Arnimallee 3, D-1 Berlin 33

A: 15.6 .81 /IUPAP

12-13 Aug

Symposium on Neutron Diffraction

Argonne, USA

Argonne National Laboratory,

9700 South Cass Ave., Argonne, IL 60439

16-22 Aug.

Vancouver, Canada 28th Congress: International Union of Pure and Applied Chemistry

IUPAC Secretariat, Bank Court Chambers, 2-3 Pound Way, Cowley Centre, Oxford OX4 3YF

16-25 Aug.

Ottawa, Canada

General Assembly of $1 \mathrm{UCr}$

2th Int. Congress of Crystallography

Charbonneau, Executive Secretary,

12th IUCr Congress, National Research Council of

Canada, Ottawa, Ontario, Canada, K1A OR6

17-20 Aug.

Québec, Canada

6th Int, Conf, on Fast lon Beam Spectroscopy

.J. Knystantas, Dépt. de Physique, Université

Laval, Québec, Canada GIK 7P4

$A b: 1.5 .81$ / IUPAP

17-21 Aug

University Park, PA, USA

5 th Int. Meeting on Ferroelectrics (IMF 5)

R.E. Newnham, Materials Res. Lab.

Pennsylvania State Univ., University Park,

PA 16802, USA

$A b: 15.281 /$ IUPAP

17-21 Aug

Uppsala, Sweden

5th Specialized Colloque Ampère: NMR in Solids

J. Tegenfeldt, Inst. of Chemistry, University of

Uppsala, Box 531, S-75121 Uppsala

A: 1.6.81 / Ab: 1.4.81 / NP / 100 / sKr. 300. -

17-21 Aug.

Bern, Switzerland

44th Annual Meeting of the Meteoritical Society

. Eberhardt, Physikalisches Institut, University of Bern Sidlerstr. 5, CH-3012 Bern

17-22 Aug.

Uppsala, Sweder

8th AIRAPT Int. Conf.: High Pressure in Research and Industry

C.-M. Backman, Inst. of Physical Chemistry,

300 Box 532, S.75121 Uppsala

17-28 Aug.

Hamburg, FRG

3rd Scientific Assembly: Int. Assoc, of Meteorology and Atmospheric Physics

V.E. Derr, Nat. Oceanic and Atmospheric Administra. tion, 325 S. Broadway, Boulder, CO 80303 , USA

19-26 Aug.

Los Angeles, CA, USA

16th Low Temperature Physics Conference (LT 16)

R. Orbach, Physics Dept., University of California, Los Angeles, CA 90024 IUPAP

23-29 Aug.

Mexico City, Mexico

th Int. Biophysics Congress of International Union for Pure and Applied Biophysics

S. Estrada-O., Director de Ciencias Biologicas y de Salud, Universidad Autonoma Metropolitana Mexico City

24-27 Aug. -

4th European Workshop on White Dwarts 5 place Janssen, F-92190 Meudon

24-29 Aug.

Trieste, Italy

Workshop on Nonlinear Evolution Equations, Solitons and Spectral Methods

A. Degasperis, Istituto di Fisica, Università di Roma Piazzale Aldo Moro 2. 1.00185 Roma

24-29 Aug.

Bonn, FRG

1981 Symposium on Lepton and Photon Interactions at High Energies

W. Paul, Physikalisches Inst. der Universitât Bonn UPAP Nussallee 12, D-5300 Bonn

24-29 Aug.

Uppsala, Sweden

8th Annual Meeting of European Geophysical Society

C.E. Lund, Inst. of Solid Earth Physics,

Uppsala University, Box 556, S-75122 Uppsala

$\mathrm{Ab}: 1.6 .81$

25-28 Aug.

Chester, UK

A tational Science

P.G. Burke, Daresbury Lab.

Warrington WA4 4AD

A : $\mathbf{1 5 . 5 . 8 1 / P P / 2 0 0 / f ~ 5 0 . ~ - ~ i n c l . ~ p r o c . ~}$ f 25 . - students

27-31 Aug

Idyllwild, CA, USA

New Problems in Low Temperature Physics

H. Bolzer, Physics Dept., Univ. of Southern California, UPAP

27 Aug. - 7 Sept.

Varenna, Italy

Course and Workshop on Plasma Astrophysics

E. Sindoni, Istituto di Fisica,

Via Celoria, 16, 1-201 3 Milano 


\section{_Physics of Atoms}

I. I. Sobelman

\section{Atomic Spectra and Radiative Transitions}

1979. 21 figures, 46 tables. XII, 306 pages (Springer Series in Chemical Physics, Vol. 1) Cloth DM 59,-; approx. US \$ 31.00

ISBN 3-540-09082-7

"... this book presents a wealth of information about the quantum mechanics of free atoms... it is nearly a must."

"... is an essential book for any science library and a very valuable text for any specialist in atomic physics..."

Contemporary Physics Applied Optics

W. Demtröder

\section{Laser Spectroscopy}

Basic Concepts and Instrumentation

1981. 431 figures, approx. 9 tables. Approx.

450 pages

(Springer Series in Chemical Physics, Vol. 5)

Cloth DM 69,-; approx. US $\$ 36.30$

ISBN 3-540-10343-0

This textbook is an introduction to modern techniques and instrumentation in laser spectroscopy. After an elementary discussion of basic subjects such as absorption and dispersion of light, coherence, line broadening effects and saturation phenomena a detailed outline of spectroscopic instrumentation, such as spectrographs, interferometers and photodedectors is given.

\section{Coherent Nonlinear Optics}

\section{Excitation of Atoms and Broadening of Spectra Lines}

1981. 34 figures, 40 tables. X, 315 pages (Springer Series in Chemical Physics, Vol.7) Cloth DM 75,-; approx. US $\$ 39.40$

ISBN 3-540-09890-9

Here is a systematic treatment of the theory of elementary processes, responsible for the excitation of atomic spectra and for spectral line broadening. In dealing with the theory of spectral line broadening, the main emphasis is on problems of contemporary research, such as high-resolution laser spectroscopy, laser frequency and wavelength measurements, plasma physics, and astrophysics.
Recent Advances

Editors: M.S. Feld, V.S. Letokhov

1980. 2 portraits, 134 figures, 18 tables.

XVIII, 337 pages

(Topics in Current Physics, Vol. 21)

Cloth DM 83,-; approx.US \$ 43.60

ISBN 3-540-10172-1

Contents: M.S. Feld, V.S. Letokhov: Coherent Nonlinear Optics. - M.S. Feld, J.C.MacGillivray: Superradiance. - V.P. Chebotayev: Coherence in High Resolution Spectroscopy. - G. Grynberg, B. Cagnac, F. Biraben: Multiphoton Resonant Processes in Atoms. - C.D.Cantrell, V.S. Letokhov, A. A. Makarov: Coherent Excitation of Multilevel Systems by Laser Light. - A.Laubereau, W. Kaiser: Coherent Picosecond Interactions. - M.D.Levenson, J.J.Song: Coherent Raman Spectroscopy.

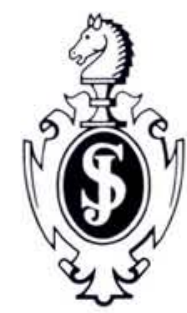

\section{Springer-Verlag Berlin Heidelberg NewYork}


31 Aug. - 3 Sept.

Bloomington, IN, USA

Int. Conf, on Phonon Physics

W.E. Bron, Dept. of Physics, Indiana University. Bloomington, IN 47401

Ab: 15.3 .81 / IUPAP

31 Aug. - 4 Sept.

Balatonfüred, Hungary

Amorphous Systems Investigated by Nuclear Methods

D.L. Nagy, Central Research Institute for Physics, POB 49, H-1525 Budapest

A: 31.5 .81 / Ab: $31.3 .81 /$ PP: $31.5 .81 / 150$ / inv. / US $\$ 95 .-$

31 Aug. - 4 Sept.

Canterbury, UK

10th Int. Colloquium:

Group-Theoretical Methods in Physics

L.L. Boyle, University Chemical Laboratory, University of Kent, Canterbury CT2 7NH

A: 20.6.81 / PP: $31.5 .81 / 150 / £$ 48. - incl. proc.

31 Aug. - 5 Sept.

Graz, Austria

Congress and 12 th Assembly of the International Commission for Optics

K. Schindl, ICO 12, C. Reichert Optische Werke AG, Hernalser Hauptstr. 219, A-1170 Vienna

A: $31.5 .81 / \mathrm{Ab}: 31.3 .81 / \mathrm{PP} / \mathrm{AS} 1200$. 600. - (students)

31 Aug. - 5 Sept.

Graz, Austria

Symposium on Astronomical Optics (with ICO 12)

A. Lohmann, Physics Dept., University. Rommelstr. 1, D-852 Erlangen

September

Workshop on Nuclear Physics

International Centre for Theoretical Physics, POB 586, 1-34100 Trieste

1-3 Sept.

1-3 Sept. Bad Honnef, FRG
Elektronische Strukturberechnungen Postfach 102148, D-4630 Bochum

inv. / German

1-4 Sept.

Canberra, Australia

2nd Australian National Laser Conf.

Secr. 2ANLC, Dept. of Engng. Physics, Res. School of Phys. Sciences,

POB 4, Canberra A.C.T. 2600

$A b: 31.5 .81 / P P: 31.5 .81 / \$ 60 .-; 20 .-$ (students)

1-4 Sept.

Visegrad, Hungary

Symposium on Particle Physics: Gauge Theories and Lepton-Hadron Interactions

G. Pocsik, Dept. of Theor. Physics, Eötvös University, Puskin u. 5-7, H-1088 Budapest

A: $1.5 .81 /$ PP: $1.5 .81 / 80 /$ inv. / US $\$ 70$ - approx.

1-5 Sept.

Crimea, USSR

IAU Colloquium No 66: On the Problems of Solar and Stellar Oscillations

E. Ergma, Astronomical Council, AN USSR, Pjatniskaja 48, SU-109017 Moscow

1-11 Sept.

Udine, Italy

2nd Int. Seminar: Mathematical Theory of Dynamical Systems and Microphysics (Geometry and Mechanics

A. Blaquière, CISM, Piazza Garibaldi 18 1-33100 Udine

Ab: $15.3 .81 / \mathrm{PP}$

3-4 Sept. Nottingham, UK
Radiofrequency Spectroscopy: Recent Developments

Nottingham, UK and Future Prospects

E.R. Andrew, Dept. of Physics, Univ. of Nottingham, 200 University Park, Nottingham NG7 2RD

4-5 Sept.

Istanbul, Turkey

Seminar on Problems of Physics in the Less Developed Regions in Europe

Mehmet Tomak, Physics Dept., Middle East inv. Technical University, Ankara

4-8 Sept. -

Tokyo, Japan

22nd Colloquium Spectroscopium Internationale

9 th Int. Cont. on Atomic Spectroscopy

Japan Society for Analytical Chemistry, Gotanda Sanhaitsu, 26-2 Nishigotanda, 1-chome. Shinagawa-ku, Tokyo 141

6-12 Sept.

32nd International Astronautical Congress - Space: Mankind's Fourth Environment

Int. Astronautical Federation, 250 rue Saint-Jacques, F-75005 Paris, France
6-13 Sept.

Balatonfüred, Hungary

GIREP Conf. on Physics Education: Nuclear Physics and Nuclear Power

G. Marx, ELTE, Dept. of Atomic Physics, Puskin u 5-7 $\mathrm{H}-1088$ Budapest

$A: 31.5 .81 / A b: 31.5 .81 / 150 /$ inv. / US $\$ 80$.

7-10 Sept.

Caen, France

9th Int. Conf. on Cyclotrons and their Applications

P. Lapostolle, GANIL, B.P. No. 5027 F-14021 Caen Cedex

A: May 81 / Ab: May 81 / PP / 300

FF 300. - incl. proc.

7-10 Sept.

Cambridge, UK

Conf, of Electron Microscopy and Analysis Group (EMAG ' 81

The Meetings Officer, The Institute of Physics, 47 Belgrave Square, London SW $1 \times 80 \times$

7-11 Sept.

Grenoble, France

4th Int. Symposium on Neutron-Capture Gamma-Ray Spectroscopy and Related Topics

B. Maier, Inst. Laue-Langevin, B.P. No. 156 F-38042 Grenoble Cedex

\section{7-11 Sept.}

Istanbul, Turkey

5th EPS General Conference: Trends in Physics

PS 5, European Physical Society, POB 69, CH-1213 Petit-Lancy 2, Switzerland

PP / SFr. 140. - (IOM); 180. - (memb. EPS nat. soc.); 40.- (student); 220. - (other); incl. proc.

\section{7-11 Sept.}

Graz, Austria

ICO-12 Satellite Meeting: Optics in Biomedical Sciences

K. Schindl, ICO-12 Satellite Meeting.

C. Reichert Optische Werke AG,

Hernalser Hauptstr. 219, A-1170 Vienna

A: $31.5 .81 / \mathrm{Ab}: 31.3 .81 / \mathrm{PP}: 7.9 .81 / 150$

AS 1000.-; 500.- (students). After 31.5.81:

AS $1200 .-$ and $700 .-$ resp.

7-11 Sept. Amsterdam, NL

11th European Microwave Conference

T.G. van de Roer, EUMC 81, Eindhoven Tech. Univ. POB $513, \mathrm{NL}-5600 \mathrm{MB}$ Eindhoven

$A b: 15.3 .81$ URSI

7-12 Sept

Bristol, UK

11 th Int. Conf. on Solid State Nuclear Track Detectors

P.H. Fowler, H.H. Wills Physics Laboratory, Tyndall Ave., Bristol BS $81 \mathrm{TL}$

$A b: 30.4 .81$

8-10 Sept.

Zelenchuk, USSR

IAU Colloquium No 67

Instrumentation for Large Telescopes

V. Rylov, Special Astrophys. Observatory, Stavropolsky kraj, Zelenchuk

8-10 Sept.

Namur, Belgium

2nd Int Conf on Chemical Analysis: Microanalysis using Charged Particles, Accelerators

G. Demortier, Microanalysis Conf., LARN rue Muzet 22, B-5000 Namur

Ab: $15.4 .81 / 100 /$ bFr. 3000 . -

8-10 Sept.

Paris, France

7th Int Symposium on Microprogramming and Microprocessing (EUROMICRO 81 )

$P$. Le Beux, Université de Paris I.

place du Panthéon, F-75231 Paris Cedex 05 P: 15.3 .81

8-11 Sept. Copenhagen, Denmark

7th European Conf. on Optical Communication

Copenhagen Congress Centre, Bella Center A/S Center Boulevard, DK-2300 Copenhagen 5 $\mathrm{Ab}: 31.3 .81$

9-11 Sept.

Meeting of Particles and Fields Div. (APS

C. Heusch, Dept. of Physics, Univ. of California, Santa Cruz, CA 95060

\section{0-15 Sept.}

Pacific Grove, CA, USA

Int. Conference on Actinides

N. Edelstein, Materials and Molecular Research Division. Bldg. 70A, Lawrence Berkeley Laboratory, Univ. of California, Berkeley, CA 94720

14-15 Sept.

London, UK

1st European Conf. on Integrated Optic

IEE Conf. Dept., Savoy Place, London WC2R OBL

P: 10.4 .81
14-16 Sept.

Toulouse, France

Hertzian Optics and Dielectrics

G. Raoult, B.P. 45, F-63170 Aubière

A: May 81 / PP / Engl., French / FF 200 - approx.

14-16 Sept.

Reading, UK

Biennial Polymer Physics Conference

D.C. Bassett, J.J Thompson Physics Lab.

University of Reading, Reading RG6 2AF

14-16 Sept.

Münster, FRG

4th European Conf. on Surface Science (ECOSS 4)

A. Benninghoven, Physikalisches Institut

Univ. Münster, Domagkstr., D-4400 Münster

PP / 500

14-16 Sept.

Selectivity in Heterogeneous Catalysis Mrs. Y.A. Fish, The Royal Society of Chemistry,
Burlington House, London W1V OBN

A: Aug. $81 / \mathrm{Ab}: 31.10 .80 / \mathrm{PP}$ : May $81 / 200 /$ f 20 .-

14-17 Sept.

Chicago, IL, USA

3rd Conf. on Computation of Electromagnetic Fields

R.P. Smith, COMPUMAG Conf., Building 362

Argonne National Laboratory. Argonne, IL 60439

\section{4-17 Sept. Toulouse, France \\ European Solid State Device Research Conf. (ESSDERC '81) \\ G. Sarrabayrouse, LAAS, 7 ave. du Colonel \\ Roche, F-31400 Toulouse \\ A: Aug. 81 / Ab: 1.5.81 / P: 1.5.81/400}

14-17 Sept.

Linz, Austria

4th Int. Conf. on Physics of Narrow Gap Semiconductors

A. Lopez-Otero, Inst. f. Experimentalphysik, Johannes Kepler Univ., A-4040 Linz

A: 20.6.81 / Ab: 15.4.81 / PP: 14.7.81 / 200 / IUPAP /

AS 1200.-; 1500. - after 20.6.81; incl. proc.

14-18 Sept. Liblice, CSSR

Symposium on High Temperature Gas Dynamics

M. Pichal, Institute of Thermomechanics,

Czechoslovak Academy of Sciences,

Puskinovo nam. 9, CS-16000 Prague 6

14-18 Sept.

Paris, France

Microscopic Aspects of Adhesion and Lubrification

C. Troyanowsky, Société de Chimie Physique, 10 rue Vauquelin, F-75231 Paris Cedex 05

Ab: 31.1 .81 / PP: 1.9.81 / FF 500.- approx.

14-18 Sept.

Suzdal, USSR

1st Int. Conf. on Measurement Problems in Medicine and Biology (IMEKO)

NTO PRIBORPROM

Pr. Marxa 17, 121019 Moscow G-19

14-18 Sept.

Reading, UK

High Resolution Molecular Spectroscopy

I.M. Mills, Dept. of Chemistry, University of Reading. Whiteknights, Reading RG6 2AD

E. 30 . - approx.

14-19 Sept.

10th European Conf. on Controlled Fusion and Plasma Physics

M.S. Rabinovich, Lebedev Physical Institute, Leninsky prospect 53

SU-117924 Moscow

A: $\mathbf{1 . 9 . 8 1 / A b : 2 0 . 2 8 1 / P P : ~ 5 . 5 . 8 1 / 6 0 0 ~ / ~}$ Engl., Russ. / Rbl. 105. - (IOM) 135. (others); 50.- (students); incl. proc.

15-18 Sept.

Ferrara, Italy

10th European Conf. on Few- and Several-Body Problems in Nuclear Physics

L. Lovitch, Istituto di Fisica, Università di Ferrara,

Via Paradiso $12,1.44100$ Ferrara

A: $30.4 .81 / \mathrm{Ab}: 31.5 .81 / 120 /$ Lit. 20000 .-

16-22 Sept.

Cozumel, Mexico

AU Symposium No 99: Wolf-Rayet Stars, Obser. vations, Physics and Evolution

C. De Loore, Astrophysical Inst, Vrije Universiteit Brussel, Pleinlaan 2, B-1050 Brussels, Belgium

Ab: $31.3 .81 / \mathrm{PP} / 100 /$ US $\$ 30 .-$ approx

17-18 Sept.

Münster, FRG

Oberflächenphysik (DPG

. Müller, Lehrstuhl f. Festkörperphysik,

Univ. Erlangen-Nürnberg.

Erwin-Rommel-Str. 1, D-8520 Erlangen

Ab: 15.6 .81 


\section{Europhysics Journals from Taylor \& Francis}

\section{Advances in Physics}

A journal in which the latest advances in condensed-state and molecular physics are reviewed by active physicists in these specialised fields. The editorial board invites contributions from research groups world-wide, and new findings are placed in the context of emerging frameworks of understanding.

Bi-monthly. $\quad$ 880.00, \$240.00, DM352.00

\section{Contemporary Physics}

The journal that enables physicists in a wide variety of occupations - research, industry, teaching - to keep abreast of developments in physics. All articles are specially commissioned from distinguished authors worldwide and will be of great value to under-and postgraduate students seeking coherent and up-to-date explanations of currently important subjects. Bi-monthly. £46.00, \$145.00, DM202.00

\section{Molecular Physics}

An international journal at the interface between chemistry and physics. It covers the physics of molecules and in particular molecular structure, properties, dynamics and collisions and the equilibrium, transport and relaxation properties of molecular assemblies. 1980 saw the journal expanded to eighteen issues per year. 18 issues $£ 250.00, \$ 670.00, D M 1100.00$

\section{Optica Acta}

Publishes papers on classical and modern optics, and accepts papers both on theory and on the development of techniques and advanced instrumentation. Short reports giving advance notices of original results are published especially quickly. Optica Acta is published in association with the Institute of Physics and the International Commission for Optics. Monthly. $\quad$ 1115.00,\$340.00, DM508.00

\section{Philosophical Magazine, A and B}

For over 180 years, Philosophical Magazine has published fundamental research into 'natural philosophy'. Today the journal covers the whole field of condensed matter physics and takes especial care to add new areas to its coverage as they arise. Part A - defects and mechanical properties of condensed matter, Part B - electronic, optical and magnetic properties of condensed matter. Monthly in 2 parts. $\quad £ 165.00, \$ 470.00, D M 724.00$

N.B. A combined subscription with Advances in Physics is available.

Sample copies and instructions for authors are available on request.

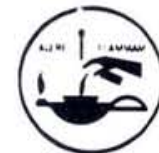
Taylor \& Francis Ltd 4 John Street, London WC1 2ET.
20-23 Sept. Kanagawa, Japan Int. Symposiun pounds

H. Yanal, Dept. of Electronic Engng. Univ. of Tokyo, Bunkyo-ku, Tokyo 113

21-23 Sept. Slupsk, Polan 2nd Maritime Ultra-Microtrace Spectrometry Conf. IMUMS

H. Szmacinski, MUMS Conf., Dept. of Physics, Pedagogical University, ul. Arciszewskiego 22 PL-76-200 Slupsk

A: 28.2.81/Ab: 28.2.81/Engl., Polish

21-24 Sept.

Brit. Assoc. of Crystal Growth (BACG-CBF Celebratory Meeting (1951-81)

D.J. Stirland, Plessey Research (Caswell) Ltd. Allen Clark Research Centre, Caswell. Towcester, Northants NN12 8EO

A: July 81

21-25 Sept.

th Int. Thin Films Congress

Herzlia-on-Sea, Israe Thin Films, Properties and Devices

. Shapira, 5th Int. Thin Films Congress, POB 3054, Tel Aviv

A : 21.9.81 / Ab: 15.4.81 / PP / 350 / IUPAP / US $\$ 75 .-; 25$. - students

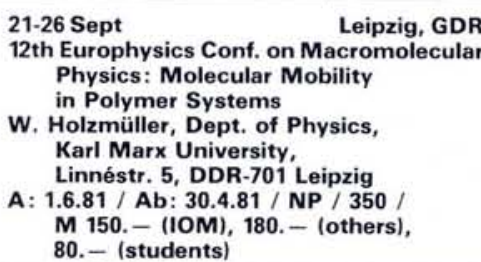

L. Alte da Veiga, Dept. de Fisica, Universidade de Coimbra P. 3000 Coimbra

A: 30.6.81/Ab: $30.4 .81 / \mathrm{NP} /$ Portuguese

\section{2-24 Sept}

7 th ESSCIRC

Univ, of Dortmund, Postfach 500500 D-4600 Dortmund 50

$A b: 20.4 .81$

\section{2-25 Sept.}

Symposium on Computerized Measurement

Yugoslav Assoc. of Measurement, Control and

Automation, JUREMA, POB 398, YU-41001 Zagreb Ab: $10.9 .80 / P: 31.1 .81 /$ US $\$ 80$.- or Yu. Din. 1600 .

\section{2-25 Sept. \\ Soft Magnetic Materials (SMM 5) \\ Brissonneau, Lab. d'Electronique, ENSIEG, Domaine Universitaire, B.P. 46, F-38402 St. Martin d'Hères \\ Ab: 1.3.81 / PP: 15.5.81/250 / FF 600. - (IOM); 700. - (others): 70. - (students)}

\section{2-25 Sept.}

6th Symposium on the Jahn-Teller Effect
Miss D. van der Wey, Phys. Chemistry,

Katholieke Universiteit, Toernooiveld

NL-6525 ED Nijmegen

23-25 Sept.

Vienna, Austria

Annual Meeting of the Austrian Physical Society

H. Warhanek, Inst. f. Experimentalphysik,

Strudlhofgasse 4, A-1090 Wien

NP / German

23-25 Sept.

5 th National Quantum Electronics Cont.

Hull, UK

D.R. Hall, Dept. of Applied Physics,

University of Hull, Hull HU6 7RX

A: $31.7 .81 / \mathrm{Ab}: 30.6 .81 / \mathrm{PP}$

25-26 Sept.

Davos, Switzerland

Herbsttagung: Schweizerische Physikalische Ges.

$\mathrm{H}$. Beck, Institut de Physique

rue Breguet 1, $\mathrm{CH}-2000$ Neuchâtel

Ab: $1.8 .81 / \mathrm{PP} / 100 /$ Engl., French, German
28 Sept. - 2 Oct.

Nice, France

SEAS Anniversary Meeting: Workshop on Analysis and Design of Computer Installations and Communication Systems

C.J.M. Aarts, SEAS HQ, Toernooiveld,

NL 6525 - ED Nijmegen

A : $1.9 .81 / \mathrm{Ab}: 15.5 .81 / \mathrm{PP}: 1.9 .81 / 250 / \mathrm{hFL} .700$, -

@ 30 Sept. - 7 Oct.
Europhysics Study Conf. on Dynovnik, YU
Processes at Surfaces (Atomic Spec-
troscopy of Surfaces)
Marijan Sunjic, Fizicki Zavod,
Marulicev trg 19, YU-41000 Zagreb
100 / US $\$ 350 .-$ incl. board;
$300 .-$ (students)

1-7 Oct.

Moscow/Yerevan, USSR

Int. Symposium on Chemical Physics (IUPAC)

N.M. Emmanuel, Institute of Chemical Physics,

Academy of Sciences of USSR, Vorobyevskoye

Chaussee 2-6, SU-117334 Moscow

4-9 Oct.

Bad Honnef, FRG

Int. Symposium on Polarization Phenomena

G. Graw, Sektion Physik, Universität München, Am Coulombwall 1, D-8046 Garching

5-7 Oct.

Aussois, France

3rd Symposium on Frequency Standards and Metrology

C. Audoin, Lab, de l'Horloge Atomique, Bât. 221 Université Paris-Sud, F-91405 Orsay Cedex

6-8 Oct.

Lausanne, Switzerland

Int. Conf. on Modern Filtering Techniques

Sec., Journées d'électronique, Dépt. d'électricité, EPFL chemin de Bellerive 16, $\mathrm{CH}-1007$ Lausanne

$\mathrm{Ab}: 1.4 .81 / \mathrm{PP}: 1.8 .81 /$ French, Engl.

6-8 Oct.

Hamburg, FRG

4. Symposium d. Deutschen Arbeitsgemeinschaft fü Mustererkennung: Modelle und Strukturen

B. Radig, Fachbereich Informatik, Universität Hamburg, Schlüterstr. 70, D-2000 Hamburg 13

Ab: $15.4 .81 /$ German

6-14 Oct.

Erice, Italy

Europhysics Study Conf.: Unification of Fundamental Interactions - II

S. Ferrara, TH Div., CERN, CH-1211 Geneva 23 
7-9 Oct.

Paris, France

4th Conf. on Numerical Methods in Fluid Mechanics

H. Viviand, 29 ave. de la Division Leclerc, F-92320 Châtillon

$A b: 15.3 .81$

\section{2-15 Oct.}

Bad Honnef, FRG

Resonances in Heavy lon Reactions

K. Eberhard, Sektion Physik, Universität München, inv Am Coulombwall 1, D-8046 Garching

\section{2-15 Oct.} Berlin (West)

50 Years Dynamics of Chemical Reactions

J.P. Toennies, MPI f. Strömungsforschung, Böltinger Str. 6-8, D-3400 Göttingen

PP / $150 /$ inv.

\section{4-16 Oct.}

Karlovy Vary, CSSR

Temperature Measurement in Industry and Science IMEKO Secretariat, POB 457, H-1371 Budapest

$A b: 1.9 .80 / P: 31.3 .81$

\section{5-17 Oct.}

Schenectady, NY, USA

IAU Colloquium No 68: Astrophysical Parameters for Globular Clusters

A.G. Davis Philip, Dudley Observatory,

69 Union Ave., Schenectady, NY' 12308

19-23 Oct.

Dubrovnik, Yugoslavia

6th European Regional Astronomy Meeting: Sun and Planetary Systems

G. Teleki, Astronomical Observatory, Volgina 7. YU-11050 Belgrade

PP / 500 / IAU, IUGG / US \$50. - approx

\section{0-23 Oct.}

Bad Honnef, FRG

Fachtagung Magnetooptik

W. Wetting, Fraunhofer-Institut $f$. Angewandte Festkörperphysik, Eckerst. 4, D-7800 Freiburg inv. / German

\section{1-23 Oct.}

San Francisco, CA, USA

Nuclear Science Symposium

P.L. Phelps, Jr., Lawrence Livermore Lab., POB 5507, L-453, Livermore, CA 94550

22-24 Oct. Bloomington, IN, USA Workshop on Pion Production and Absorption in Nucle R.D. Bent, Milo B. Sampson Lane, Bloomington, iN 47405

\section{6-30 Oct.}

Kissimmee, FL, USA

Annual Meeting: Optical Society of America

J.W. Quinn, Optical Society of America, Executive Office, Suite 200, L Street N.W. Washington, DC 20036

$A b: 26.6 .81 /$ US $\$ 75$. -

27-29 Oct. Brunn a/Gebirge, nr. Vienna, A 2nd Joint Meeting: Vacuum Div. of Eötvös Lorand Phys. Soc. and Austrian Vacuum Soc.

R. Dobrozemsky, Austrian Vacuum Society, Inst. Allgem. Physik, TU Vienna, Karlsplatz 13, A-1040 Vienna

\section{8-30 Oct.}

Asilomar, CA, USA

Meeting of Nuclear Physics Division (APS)

The American Physical Society, 335 East 45th Str., New York, NY 10017

2-6 Nov. New York, USA

Meeting of Plasma Physics Division (APS)

The American Physical Society, 335 E. 45th Street, New York, NY 10017

\section{9-12 Nov.}

Argonne, IL, USA

Int. Meeting on Neutron Irradiation Effects in Solids

Th. H. Blewitt, Materials Science Div., Bldg 212, 9700 S. Cass Ave., Argonne, IL 60439

9-13 Nov. Bad Honnef, FRG Young European Radioastronomers' Conference (YERAD)

R. Schwarz, MPI für Radioastronomie, Auf dem Hügel 71, D-5300 Bonn

10-13 Nov. Atlanta, GA, USA

27th Annual Conf. on Magnetism and Magnetic Materials

H.C. Wolfe, American Institute of Physics, 335 East 45th Str., New York, NY 10017 $\mathrm{Ab}: 20.7 .81 / \mathrm{PP} / \mathrm{US} \$ 70$. -

15-20 Nov.

Joint Meeting: American Assoc. of Physicists in Medicine, Radiological Soc. of N. America

Ms. A. Swenson, Radiology Soc. of N. America, Suite 1150, Oak Brook Regency Towers 1415 W. 22nd Str., Oak Brook, IL 6052
15-22 Nov.

Erice, Italy

Europhysics Study Conf.: The Search for Charm, Beauty and Truth at High Energies

G. Bellini, Istituto di Scienze Fisiche,

Via Celoria 16, I-20133 Milano

17-19 Nov.

London, UK

2nd Int. Symposium on Technical Diagnostics

IMEKO Secretariat, POB 457, H-1371 Budapest 5

A: 19.11.80/PP: 29.5.81/Engl., Fr., German, Russ.

\section{2-24 Nov.} Monterey, CA, USA

Meeting of the Fluid Dynamics Division (APS

The American Physical Society, 335 East 45th Street. New York, NY 10017

23-25 Nov.

New Orleans, LA, USA

APS Fall Meeting

W.W. Havens Jr., APS, 335 E. 45th Street, New York, NY 10017

$\mathrm{Ab}: 4.9 .81$

2-4 Dec.

Dresden, GDR

Barkhausen Anniversary Colloquium on Components and Systems of Electrons

W. Cimander, Techn. Univ. Dresden,

Sektion Informationstechnik

Mommsenstr. 12, DDR-8027 Dresden

Ab: $10.10 .80 /$ German / M 125

New York, USA

Meeting of Electron and Atomic Phys. Div. (APS)

The American Physical Society, 335 E. 45th Street, New York, NY 10017

7-12 Dec.

Miami Beach, FL, USA

6 th Int. Conf. on Infrared and Millimeter Waves

K.J. Button, MIT National Magnet Laboratory,

170 Albany Street, Cambridge, MA 02139

$\mathrm{Ab}: 30.7 .81$ / PP / URS

\section{6-17 Dec.}

Structure of the Interfacial Region

Oxford, UK

Mrs. Y.A. Fish, The Royal Society of Chemistry, Burlington House, London W1V 0BN

A: Nov. 81 / Ab: 15.12.80 / PP: 15.8.81 / $100 /$ f 20. -

\section{2}

Europhysics Conference on Molecular Magnetism Poland

B Jezowska-Trzebiatowska, Institute of Chemistry University of Wroclaw, 14 Joliot-Curie Street, PL-50-383 Wroclaw

\section{5-28 Jan.}

San Francisco, CA, USA

General Meeting: American Physical Society

American Physical Society, 335 E. 45th Street, New York, NY 10017

20-26 Feb.

San Francisco, CA, USA

COMPCON Spring 1982

H. Hayman, POB 639, Silver Spring, MD 20901

\section{8-12 March}

Dallas, TX, USA

General Meeting: American Physical Society

American Physical Society, 335 E. 45th Street,

$$
\text { New York, NY } 10017
$$

22-25 March

Manchester, UK

General Conference of EPS Condensed Matter Division The Meetings Officer. The Institute of Physics 47 Belgrave Square, London SW $1 \times 80 X$

22-26 March

Nottingham, UK

Int. Conf. on Electron Spin Resonance of Organic and Bio-organic Radicals

J.B. Raynor, Chemistry Dept., The University, Leicester, LE1 7RH

\section{2-27 March}

Grenoble, France

3rd Joint Varenna - Grenoble Int. Symposium on Heating in Toroidal Plasmas

C. Gormezano, Fusion-SIG, Centre d'Etudes Nucléaires, 85X, F-38041 Grenoble Cedex

\section{9-31 March}

Vacuum ' 82

The Meetings Officer, The Institute of Physics,

47 Belgrave Square, London SW $1 \times 80 X$

4-8 April

Arlington, TX, USA

6th Int. Conf. on Positron Annihilation

L.M. Diana, Box 19059, Univ. of Texas,

PP

5-7 April

Van der Waals Molecules Mrs. Y.A. Fish, The Royal Society of Chemistry,
Burlington House, London W1V OBN

A: March 82 / Ab: $31.5 .81 /$ PP: $15.12 .81 / 200$

13-16 April

Zurich, Switzerland

Int. Conference on Valence Instabilities

P. Wachter, Laboratorium f. Festkörperphysik ETH Zurich, CH-8093 Zurich

Ab: 10.1.82 / $200 /$ incl. proc. SFr. 150. - (IOM) 180. - (others)

14-16 April

Phoenix, AZ, USA

Conference on Lasers and Electro-Optics (CLEO)

J.W. Quinn, Optical Society of America,

1816 Jefferson Place, N.W., Washington, DC 20016

\section{4-16 April}

Graz, Austria

Europaische Rheologentagung

Kirschke, Deutsche Rheologische Gesellschaft, Unter den Eichen 87, D-1000 Berlin 45

A: April 82 / PP: Autumn $81 / 300$

20-23 April

Durham, UK

Int. Conf. on II-VI Compounds

B. Lunn, Dept. of Physics, The University, PP Hull HU6 7RX

3-7 May

Gatlinburg, TN, USA

5th Int. Conf. on Plasma Surface Interactions in Controlled Fusion Devices

R.A. Langley, Oak Ridge Nat. Lab., POB Y, Oak Ridge, TN 37830

\section{4-6 May}

San Diego, CA, USA

27th SAMPE Symposium and Exhibition

Marge Smith, SAMPE, POB 613, Azusa, CA 91702

$A b: 1.8 .81 / P: 1.1 .82$

10-13 May

Budapest, Hungary

SEAS Meeting

C.J.M. Aarts, SEAS Headquarters Toernooiveld, NL 6525 ED Nijmege

A: $15.4 .82 / \mathrm{Ab}: 15.10 .81 / \mathrm{PP}: 15.11 .81$

\section{7-19 May}

Ottawa, Ontario, Canada International Conference on Plasma Science

John Alcock, National Research Council, Ottawa, Ontario K1A OR6

\section{7-22 May}

Ottawa, Canada

Int. Symposium on Solar Terrestrial Physics

J.G. Roederer, Geophysical Institute,

University of Alaska, Fairbanks, Alaska 99701, USA

IUGG, IUPAP, IAU, COSPAR, URSI, SCOSTEP

\section{7-29 May}

Ottawa, Canada

24th COSPAR Plenary Meeting and Associated Activities

Z. Niemirowicz, Exec. Secretary, COSPAR, 51 boulevard de Montmorency, F-75016 Paris

Ab: 15.1 .82 / PP / 800 / Engl., French / COSPAR / US $\$ 95 .-$

23-26 May

Damp 2000 nr. Kiel, FRG

th Int. Conf. on Photon Correlation Techniques in Fluid Mechanics

E.O. Schulz-DuBois, Inst. f. Angewandte Physik, Universität Kiel, Olshausenstr. 40-60, D-2300 Kiel 1

PP / 120 / incl. proc. DM 180. - (IOM): 200. - (others); 120. - (students)

\section{4-28 May}

9th IMEKO Congres

IMEKO Secretariat, POB 457, H-1371 Budapest

Ab: $31.3 .81 / P: 31.1 .82$

1-5 June Ottawa, Ontario Canada 
4 th international Congress on Quantum Chemistry

J.L. Calais, Quantum Chemistry Group, University of Uppsala, Box 518, S-751 20 Uppsala

30 June - 2 July

Oxford, UK

ath Annual Conf, on Plasma Physics

C.N. Lashmore-Davies, Culham Laboratory GB-Abingdon OX14 3DB

12-16 July

Amherst, MA, USA

1982 IUPAC Macromolecular Symposium Engineering, University of Massachusetts. Amherst, MA 01003

12-17 July Paris, France

21st Int Conference on High Energy Physics

Conf. Secretariat, 20 rue Berbier du Mets, UPAP F-75013 Paris

20-23 July Montreal, Quebec, Canada Joint Intermag and Magnetism and Magnetic Materials Conference

A. Yellon, Ecole Polytechnique, University of Montreal Montreal, Quebec, Canada H3C 3 A7

27-30 July

Liège, Belgium

14th EGAS Conference

N. Grevesse, BFS-Group, University of Liège,

250 Sart Tilman B15, B-4000 Liège

August

Besancon, France

AU Symposium: Internal Kinematics and Dynamics of Galaxies

M. Crézé, Observatoire de Besançon, F-25000 Besançon

17-24 Aug.

Hamburg, FRG

10 th Int. Congress on Electron Microscopy

German Convention Service, Dammtorstr. 12

D-2000 Hamburg 36

17-26 Aug.

Patras, Greece

18th General Assembly of Int. Astronomical Union

Mrs. B. Manning, IAU Secretariat,

61 ave de I'Observatoire, F-75014 Paris

US $\$ 100-; 120-$ (after 31.5 .82

23-27 Aug.

Poznan, Poland

22 d Int. Ampère Congress: Magnetic Resonance and Related Phenomena

J. Stankowski, Instytut Fizyki Molekularnej

A: 4.6.82 / Ab: 15.9.81 / PP: 1.5.82 / English /

US $\$ 100$.

23-27 Aug.

Leeds, UK

9th Annual Meeting of European Geophysical Society

J.C. Briden, Dept. of Earth Sci

University of Leeds, Leeds LS2 9JT

Ab: 1.6 .82

25-30 Aug.

Nikko, Japan

Sagamore VII Conf. on Charge, Spin and Momentum Densities

Y. Saito, Institute for Solid State Physics, Univ. of Tokyo, 22-1 Roppongi 7-chome, Minato-Ku, Tokyo 106

27-30 Aug.

Lake Kawaguchi, Japan

2nd Int. Symposium on Molecular Beam Epitaxy and Related Clean Surface Techniques

MBE CST-2, R. Ueda, Dept. of Applied Physics, School of Science and Engineering, Waseda University, 3-4-1, Ohkubo, shinjuku-ku, Tokyo
J.C.W. Chien, Department of Polymer Science and

ul. Smoluchowskiego 17/19, PL-60-179 Poznan

\section{Journal of Physics B: Atomic and Molecular Physics}

\section{A Europhysics Research Journal}

A journal of major international importance on the structure and interactions of atoms and simple molecules.

It publishes papers and letters covering the following subjects:

* Electronic, atomic and molecular collisions

* Atomic and molecular structure and spectroscopy

* Also those aspects of spectroscopy, surface physics, astrophysics, plasma, discharge maser and laser physics, quantum optics, nonlinear optics and other investigations where the objects of study are the elementary atomic, ionic or molecular properties or processes.

\section{Why not inspect a copy, free of charge?}

Write to: Marketing Department, Room 184

The Institute of Physics

Techno House, Redcliffe Way

Bristol BS1 6NX, England
29 Aug. - 3 Sept.

7 th European Crystallographic Meeting

ECM-7, J. Bernstein, Dept. of Chemistry,

Ben Gurion Univ. of the Negev, Beer Sheva 84120

30 Aug.-3 Sept.

Dublin, Ireland th Europhysics Topical Conference on Lattice Defects in Ionic Crystals

J. Corish, Dept. of Chemistry, University College Dublin, Belfield, Dublin 4

A : 30.4.82/Ab: 28.2.82 / PP: 28.2.82/ 250 /

incl. proc. approx. IR $€ 60 .-; 20 .-$ (students)

30 Aug. - 3 Sept.

Vienna, Austria

9th Int. Mass Spectrometry Conference

Interconvention, POB 105, A-1014 Vienn

Baden, Austria 6th Int. Workshop: Rare Earth - Cobalt Permanent

3rd Int. Symp. on Magnetic Anisotropy and Coercivity in Rare Earth Transition Metal Alloys (3 Sept.)

J. Fidler, Inst. f. Angewandte Physik, Tech. Univ. Karlsplatz 13, A-10400 Vienna

A: $1.3 .82 / P P / 250 /$ AS 5000 .

31 Aug. - 4 Sept.

Lake Balaton, Hungary

Diffusion in Metals and Alloys

F.J. Kedves, Roland Eötvös Physical Society,

POB 240, H-1368 Budapest

A: $31.1 .82 / \mathrm{Ab}: 31.1 .82 / \mathrm{PP} / 120$

US \$100. - approx., incl. proc.

\section{September}

int. Conf, on Neutron Physics and Nuclear Data

OECD Nuclear Energy Agency.

38 blvd. Suchet, F-75016 Paris Magnets and their Applications
September

Solid State Physics under High Pressure

B. Baranowski, Inst. of Physical Chemistry,

Polish Academy of Sciences, Kasprzaka 44/52. 200 PL-01-224 Warsaw

1-3 Sept.

Oxford, UK

ESCAMPIG Conference

R.W.P. McWhirter, Rutherford Lab.

Chilton, Didcot, $0 \times 1100 X$

1-3 Sept.

Yamada Conf. on Neutron Scattering of Condensed Matter

Itoh, Inst, f. Solid State Physics, Univ, of Tokyo, 22-1 Roppongi 7-chome Minato-ku, Tokyo 106

1-8 Sept.

Baltimore, USA

Plasma Physics and Controlled Nuclear Fusion Research

R. Najar, Conference Service Section, IAEA, POB 100 , A-1400 Vienna, Austria

6-10 Sept.

Int. Conf. on Magnetism - ICM 82

Kyoto, Japan

J. Kanamori, Faculty of Science, Osaka University, Toyonaka 560

6-10 Sept.

Munich, FRG

7th Int Heat Transfer Conference

furt 97

DECHEMA, POB 970146, D-6000 Frankfurt 97

6-10 Sept.

Antwerp, Belgium

Int. Conf, on Nuclear Data for Science and Technology K.H. Böckhoff, CEC, JRC, CBNM,

B-2440 Geel, Europawijk

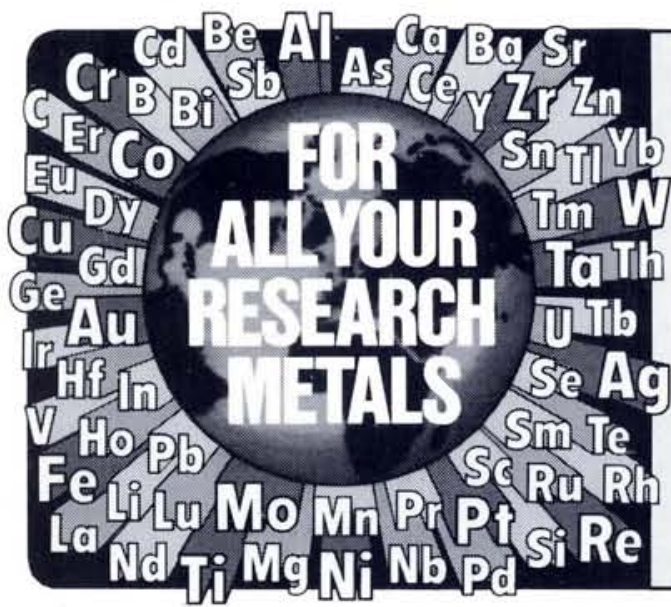

Our current catalogue lists over 2000 products including many unique or hard to get items.

\section{Foils, wires, rods, powders, tubes and compounds.}

Over 100 pure metals and alloys are available for immediate despatch, worldwide, in small quantities for research. Non-standard items made to order take a little longer.

Write, telex or telephone for your copy now !

Telephone Cambridge (0223) $\mathbf{6 9 6 7 1}$ or from abroad, dial International Access Code +44+223+69671 Telex 81683 GOODMT G

Goodfellow Metals Ltd.

Cambridge Science Park, Milton Road,

Cambridge CB4 4DJ, England

IMGOODFELLOW

MMETALS for research and industry 
6-11 Sept.

Montpellier, France

16th Int. Conf. on the Physics of Semiconductors

M. Balkanski, Université Pierre et Marie Curie, Lab. de Physique de Solides, 4 place Jussieu, Tour 13. IUPAP F-75230 Paris Cedex 05

6-17 Sept.

Varenna, italy

Course and Workshop: Diagnostics for Fusion Reactor Conditions

E. Sindoni, Istituto di Fisica, Via Celoria 16 ,

I-20133 Milano

A: 30.5 .82 / PP / US $\$ 200$. - approx.

7-9 Sept. Nijmegen, The Netherlands 4th European Study Conf. on Low Energy Molecular Collisions (MOLEC IV)

J. Reuss, Fysisch Laboratorium, Katholieke Universiteit, NL-6525 ED Nijmegen

$A b: 1.5 .82 / \mathrm{NP}: 1.2 .82 / 100 / \mathrm{hFl} .300 .-$

13-17 Sept.

Cambridge, UK

\section{The Neutron and its Application}

P. Schofield, Material Physics Div., B 521.2, AERE, 400 Harwell, Didcot, Oxon, OX11 ORA

14-16 Sept. Southampton, UK

Electron and Proton Transfer

W.J. Albery, Dept. of Chemistry, Imperial College London SW7 2AY

A: Aug. $82 /$ Ab: Oct. $81 /$ PP: May $82 /$ PP

21-24 Sept. Villafranca del Penedes, Spain 14th Europhysics Conf.: Polymer Crystals - Structure and Morphology

F.J. Balta Calleja, Instituto de Estructura de la Materia, Serrano, 119, Madrid 6

A: Jan. 82 / Ab: March 82 / SFr. 200. (IOM), 150.- (students), 250.- (others)

27 Sept. - 1 Oct. Baden-Baden, FRG 8th European Conf, on Thermophysical Properties H.E. Schmidt, Chairman 8 ETPC,

Postfach 2266, D-7500 Karlsruhe 1

6-10 Oct.

Osaka, Japan

Int. Conf. on Nuclear Physics in Cyclotron Energy Region

M. Kondo, Research Centre for Nuclear Physics, Osaka University, Yamada-Kami, Suita-shi,

Osaka 565

\section{3}

\section{Spring}

Lausanne, Switzerland

General Conference of EPS Condensed Matter Division

E. Mooser, Institut de Physique, Université de Lausanne, Dorigny, $\mathrm{CH}-1015$ Lausanne

18-21 April Toulouse, France 3rd Int. Symposium on Incoherent Light Sources

J.J. Damelincourt, Centre de Physique Atomique, Université Paul Sabatier, 118 route de Narbonne,

F-31062 Toulouse Cedex

A : 1.1.83 / PP: 30.12 .82 / FF 900. - incl. proc.

July

Zaragoza, Spain

15th EGAS Conference

E. Bernabeu, University, E-Zaragoza

8-12 August Jerusalem, Israel

7 th Int. Conf, for Vacuum Ultraviolet Radiation Physics

I.T. Steinberger, Racah Institute, Hebrew University, Jerusalem

5-9 Sept.

Aachen, FRG

11 th European Conf. on Controlled Fusion and Plasma Physics

G.H. Wolf, Kernforschungsanlage Jülich, Inst. $f$. Plasmaphysik, Postfach 1913, D-5170 Jülich 1

26 Sept. - 10 Oct. Torremolinos (Malaga), Spain 5th European Meeting on Ferrolectricity EMF 5

B. Jimenez, Centro de Fisica Aplicada, "Leonardo Torres Quevedon, Serrano 144, E-Madrid 6

A: $31.5 .83 / P: 31.5 .83 / 500 /$ US $\$ 50$. - approx

\section{Schools 1981}

15-29 June

Erice, Italy

Int. School of Atomic and Molecular Spectroscopy: Collective Excitations in Solids

B. Di Bartolo, Dept. of Physics, Boston College,

Chestnut Hill, MA 02167, USA

A: 10.4 .81 / PP / $70 /$ US $\$ 600$. - all incl.

15 June - 11 Sept.

Trieste, Italy

Research Workshop in Condensed Matter Physics

International Centre for Theoretical Physics, POB 586, 1-34100 Trieste

29 June - 10 July Car
New Directions in Guided Wave Optics Cargèse, Corsica, $\mathrm{F}$

O.B. Ostrowsky, Lab. Electrooptique, Univ. de Nice, Parc Valrose, F-06034 Nice

29 June - 10 July

Cambridge, UK

Supernovae

M.J. Rees, Institute of Astronomy

Madingley Road, Cambridge CB3 OHA

29 June - 31 July Les Houches, France

Theoretical Physics Summerschool on Chaotic Behaviour in Deterministic Systems

Ecole d'été de Physique Théorique,

Côte de Chavants, F-74310 Les Houches

A: $1.3 .81 / 50 /$ FF 2350. -

30 June - 10 July

Varenna, Italy

Mechanical Thermal Behaviour of Metallic Materials

C. Bottani, Ist. Ingegneria Nucl. del Politecnico. Via Ponzio 34/3, 1-20133 Milano

A: $30.4 .81 /$ PP / 75 / Engl. French

1-15 July Erice, Italy

Int. School of Material Science and Technology: III-V Compounds and their Applications

M. Balkanski, Université de Paris Vo, Lab. de Physique des Solides, 4 pl. Jussieu, Tour 13, F-75230 Paris Cedex 05

A: $1.5 .81 / 70$ / US $\$ 450$.

\begin{tabular}{|c|}
\hline $\begin{array}{l}\text { 1-15 July } \\
\text { Int. School of Material Science and Tech- } \\
\text { nology: Dynamics of Gas-Surface } \\
\text { Interaction } \\
\text { M. Balkanski, Université de Paris Ve, } \\
\text { Lab. de Physique des Solides, } \\
4 \text { pl. Jussieu, Tour 13, } \\
\text { F-75230 Paris Cedex 05 } \\
\text { A: } 1.5 .81 / 70 / \text { US } \$ 450 .-\end{array}$ \\
\hline
\end{tabular}

13-17 July

Pheasant Run, IL, USA

Fermilab Summer School on High Energy Particle Accelerators

R. Huson, Accelerator Physics, Fermilab, POB 500 Batavia, IL 60510

14-24 July

Varenna, Italy

Positrons in Solids

A. Dupasquier, Ist. Fisica del Politecnico, Piazzale L. da Vinci 32, 1-20133 Milan

A: $30.4 .81 / \mathrm{PP} / 75 /$ Engl., French

14-28 July

Erice, Italy

Int. School of Solid - State Device Research: Science and Technology of Large - Scale Integrated Circuits

G. Soncini, CNR-LAMEL, Via de' Castagnoli 1 1-40126 Bologna

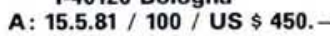

14-31 July Cargèse, Corsica, F

Summer Institute: Fundamental Interactions

Marie-France Hanseler, LPTHE, Tour 16

Univ. Pierre et Marie Curie

4 place Jussieu, F-75230 Paris Cedex 05 A: $15.3 .81 / \mathrm{PP} / 50$

\section{0-31 July}

Electron Correlations in Metals

Antwerp, Belgium

J. Devreese, U.I.A., Universiteitsplein 1.

$$
\text { B-2610 Antwerp }
$$

27 July - 7 Aug.

Stanford, CA, USA

SLAC Summer Institute on Particle Physics: The Strong Interaction

Ms. A. Mosher, SLAC, Bin 62, Stanford, CA 94305 A: $30.4 .81 / \mathrm{PP} / 300 /$ US $\$ 100$.

28 July - 7 Aug. Varenna, Italy

Data Acquisition in High Energy Physics

Mrs. G. Schwaar, EP, CERN, CH-1211 Geneva 23 A: 30.4.81/PP / 75
31 July - 11 Aug.

Erice, Italy

nt. School of Subnuclear Physics: The Unity of the Fundamental Interactions

A. Zichichi, CERN, CH-1211 Geneva 23

A: $20.7 .81 /$ SFr. 1000 .-

3 Aug. - 11 Sept. Les Houch

Les Houches, France

Ecole d'été de Physique Théorique,

Côte de Chavants, F-74310 Les Houches

A: 1.3 .81 / PP / 50 / FF 2750.-

9-29 Aug.

Dundee, UK

22nd Scottish Univ. Summer School in Physics: Magnetism in Solids, Some Current Topics

W.M. Young, Carnegie Laboratory of Physics,

University of Dundee, DD1 $4 \mathrm{HN}$

A: $15.5 .81 / 75 /$ f 275 . - approx.

10-21 Aug.

Vancouver, Canada

Relativistic Effects in Atoms, Molecules and Solids

G.L. Malli, Dept. of Chemistry and Theor. Sciences, Simon Fraser Univ., Burnaby, B.C. V5A

10 Aug. - 4 Sept.

Trieste, Italy

Physics Teaching at University Level

nternational Centre for Theoretical Physics, POB 586, 1-34100 Trieste

$50 /$ French

16-28 Aug.

Banff, Canada

Banff Summer Institute on Particles and Fields

A.N. Kamal, Dept. of Physics, Univ. of Alberta Edmonton, Alberta, Canada T6G 2JI

17-28 Aug.

Santa Barbara, CA, USA

Nuclear Theory Summer Workshop

G. Bertsch, Inst. for Theoretical Physics, University of California, Santa Barbara, CA 93106

A: $30.4 .81 / 45$

17-29 Aug.

Erice, Italy

Int. School of Quantum Electronics Physics and Applications of Integrated Optics

S. Martellucci, Engineering Faculty, Piazza V. Tecchio 80, 1-80125 Naples

A: $30.6 .81 / \mathrm{PP} / 90 /$ US $\$ 450$.

17-29 Aug.

Bad Windsheim, FRG

Quantum Optics, Measurement Theory and Test of Relativity

P. Meystre, Projektgruppe Laserforschung, Max-Planck-Gesellschaft, D-8046 Garching

A: $1.4 .81 / \mathrm{PP} / 60$

23 Aug. -4 Sept.

23 Aug. - 4 Sept. East Lansing, MI, USA East Lansing, MI 48824

23 Aug. - 26 Sept. Keilaniemi, Finland / Uppsala, S

Int. Summer Inst. in Quantum Chemistry and Solid State Physics

Pt I (23.8 - 5.9) Keilaniemi (28 part. only)

Pt II (6-26.9) Uppsala

Director, Summer Institute, Quantum Chemistry Group, Box 518, S-75120 Uppsala

A: $1.5 .81 / \mathrm{NP} / \mathrm{I}$ : sKr. 3000 .; II: 3800 .:

$1+11: 6500$.

24 Aug. - 26 Sept.

Antwerp, Belgium

Workshop III on Nonlinear Magnetodynamics

D. Callebaut, Phys. Dept., UIA, Universiteitsplein 1, B-2610 Wilrijk

25 Aug. - 5 Sept.

Poiana Brasov, Romania

Romanian Int. Summer School: Gauge Theories Fundamental Interactions and Rigorous Results

P. Dita, V. Georgescu, Central Institute of Physics, POB MG6, Bucharest - Magurele

A: $31.7 .81 / \mathrm{PP} / 140$ / Engl. / US $\$ 425$.-

29 Aug. - 9 Sept.

Erice, Italy

Int. School of Crystallography: Interfacial Aspects of Phase Transformations

B. Mutaftschiev, CRMC2 - CNRS, Campus Luminy, Case 913, F-13288 Marseille Cedex 2

A: 15.3.81 / SFr. 900.- incl. board

31 Aug. - 11 Sept.

Freiburg, FRG

Structural Elements in Particle Physics and Statistical Mechanics

M. Forger, Fakultät f. Physik, Universität Freiburg, Hermann-Herder-Str. 3, D-7800 Freiburg

A: $1.5 .81 / 75$

7-21 Sept

Manchester, UK

Postgraduate School in Nuclear Physics

J.M. Irvine, Dept. of Physics, The University, Manchester M13 9PL 
7 Sept. - 2 Oct.

Trieste, Italy

College on Microprocessors:

Technology and Applications in Physics

Int. Centre f. Theor. Phys. POB 586, 1-34100 Trieste

8-18 Sept.

Schloss Seggau, Austria

6th Ampère Summer School on Biological Applications of NMR

G.C.K. Roberts, Nat. Inst. for Medical Research, The Ridgeway, Mill Hill, London NW7 1AA, UK

A: $1.4 .81 / 120 /$ AS 3000

14-25 Sept.

Vulcano, Italy

Comparative Study of the Planets

M. Fulchignoni, Lab. di Astrofisica Spaziale, CNR, Viale dell' Università, 11, 1-00185 Roma

Sept. (2nd half) nr. Gdynia, Poland 3rd Int. School on Semiconductor Optoelectronics

M.A. Herman, Instytut Fizyki PAN, Al. Lotnikow 46, PL-02-668 Warsaw

English / Zl. 2000 - incl. proc.

16-25 Sept.

Kupari-Dubrovnik, YU

Int. Summer School of Biophysics:

Supramolecular Structure and Function

Greta Pifat, Ruder Boskovic Inst., POB 1016, YU-41000 Zagreb

A: 15.5 .81 / US $\$ 300$. -

20 Sept. -4 Oct.

Kupari-Dubrovnik, YU

Int. School of Elementary Particle Physics (17)

$\mathrm{H}$. Braun, Centre de Recherches Nucléaires,

F-67037 Strasbourg Cedex

A: $15.5 .81 / \mathrm{PP} / 90 / \mathrm{SFr} .700$.-

27 Sept. - 3 Oct.

Bad Liebenzell, FRG

KFK Summer School on Nuclear Physics: Quarks and Nuclear Forces

Mrs. V. Lallemand, Inst. f. Kern- und Teilchenphysik I, Postfach 3640 , D-7500 Karlsruhe 1 $\mathrm{PP} / 45$

28 Sept, -3 Oct.

Dubrovnik, YU

1st IMEKO Summer School: Measurement Training Application of Microcomputers in Measurement

JUREMA Secretariat, POB 398, YU-41001 Zagreb

30 Sept. - 12 Oct.

Baddock, Canada

Physics of Ion-Ion and Electron-Ion Collisions

F. Brouillard, Inst. de Physique, Science 7. B-1348 Louvain-la-Neuve

4-16 Oct.

4-16 Oct.
Int. School of Particle Accelerators: Italy RF Superconductivity (E. Picasso)

H. Lengeler, EF, CERN, CH-1211 Geneva 23

20 Oct. - 11 Dec.

Trieste, italy

ICTP Autumn Course on Variational Methods in Analysis and Mathematical Physics

International Centre for Theoretical Physics. POB 586, 1-34100 Trieste

15-29 Nov.

Erice, Italy

Int. Workshop on Classical and Quantum Metrology and Fundamental Physical Constants

P.H. Cutler, Dept. of Physics, 104 Davey Lab. Pennsylvania State Univ., University Park, PA 16802, USA

22-29 Nov.

School of Logic and Scientific Methodology Chance and Order in Natural Sciences

E. Agazzi, Istituto di Filosofia

Piazza S. Sabina 2, 1-16124 Genova

\section{2}

10-14 Aug.

Vienna, Austria

Int. School on Electron Microscopy of Magnetic Materials

J. Fidler, Inst. f. Angew. Phys., TU Vienna,

Karlsplatz 13, A-1040 Vienna

A: 1.3 .82 / PP / 120 / AS 5000 - all incl.

16-28 Aug.

Dronten, The Netherlands

Int Summer School on Nuclear Structure

P. de Witt Huberts, NIKHEF-K, POB 4395 NL-1009 AJ Amsterdam

\section{5-18 Sept.}

CERN School of Physics

Cambridge, UK

Miss D.A. Caton, Scientific Conference Secretariat, CERN, CH-1211 Geneva 23, Switzerland
ETTORE MAJORANA INTERNATIONAL CENTRE FOR SCIENTIFIC CULTURE Erice - SICILY, 16-28 Nov., 1981

Quantum Metrology and Fundamental Physical Constants A NATO ADVANCED STUDY INSTITUTE

A progressive and comprehensive course presenting in tutorial form but at an advanced level a coherent introduction to the basic physics necessary for understanding modern measurements of fundamental constants with applications in science and technology.

GENERAL INFORMATION may be obtained and application to attend the course should be made by writing to one of the School Directors:

Prof. P.H. CUTLER,

The Pennsylvania State University

104 Davey Laboratory, University Park

Pennsylvania 16802, U.S.A.

Tel. : $814 / 865-2832$

Prof. A.A. LUCAS,

Département de Physique

Facultés Universitaires de Namur

B-5000 Namur

Tel. : 41/229061

\section{POST DOCTORAL POSITION}

available at the ETH in Zurich, Switzerland, starting summer 1981.

The project consists of an experiment at the injector cyclotron at SIN (Swiss meson factory) to study parity violation in $p p$ and $p \alpha$ elastic scattering.

Candidates should have experience in nuclear or particle physics and an interest in the development of sophisticated experimental apparatus.

The position will be for one year with the possibility for renewal. Salary approx. \$25000 per year plus travel expenses.

Applicants should send their curriculum and references to:

Prof. J. Lang,

Laboratorium für Kernphysik,

ETH-Hönggerberg, CH-8093 Zürich, Switzerland. 


\section{Europhysics Conference Abstracts}

\author{
Abstracts of all \\ Conferences \\ listed in the \\ next column except \\ those marked * \\ - 10 Volumes - \\ - over 3000 pages - \\ are included in \\ ECA Series 5 \\ (Vol. A. appears \\ in two parts).
}

Abstracts are in form of "Pre-proceedings", one-two pages each.

\section{Volumes are despatched} to subscribers at the start of each Conference.

\section{Price for Series 5:}

SwFr. 290. -

Previous subscribers:

SwFr. 250.-

New subscribers

4+5: SwFr. 500.-

Individual volumes

may be sold after

Conference has

been held;

price on application.

\section{Published by the European Physical Society}

\section{EUROPEAN PHYSICAL SOCIETY}

\section{EPS Organized Conferences in 1981}

\section{CONFERENCES}

18-20 March *

International Conference on Excited State and Multi-resonant

Non-linear Optical Processes in Solids

Aussois, France

Dr. D.S. Chemia, Centre National d E
196 , rue de Paris. F- 92220 Bagneux

6-10 April

1st European Conference on Atomic Physics (ECAP)

Heidelberg, Federal Republic of Germany

Prof. G. zu Putlizz, Physikalisches institut der Universitat,
Philosophenweg 12, D - 6900 Heiddeberg 1

7-10 April

11th Europhysics Conference on Macromolecular Physics:

Thermal, Mechanical and Electrical Properties of Oriented Polymers Leeds, England

Miss D Mavston, The institute of Physics.
47. Belgrave Square, London SW $1 \times 80 \times$ UK

13-15 April *

3rd European Solar Meeting: Solar Activity

Oxford, England

Prot. C Jordan, Department of Theorenical Physics, University of Oxford.

9-12 June

Europhysics Conference: Nuclear and Atomic Physics with Heavy lons

Bucharest, Romania
Or. LA. Dirobantu, Central institute of Physics
POB MG.6. B. Bucharest

9-15 July

International Conference on High Energy Physics (Europhysics

Lisbon, Portugal

Conference)

Prot. J. Dias de Deus, FTMM, INIC

25-28 August

Vector and Parallel Processors in Computational Science

Chester, England

Dr. P.G. Burke. Daresbury
Warringtion WA4 $4 A D$ UK

7-11 September *

5th EPS General Conference: Trends in Physics

Istanbul, Turkey

Executive Secretary, EPS
POB $69 \mathrm{CH}-1213$ PetritLancy 2

14-17 September

European Solid State Device Research Conference (ESSDERC 81)

Toulouse, France

De. G. Sarrabarrouse, LAAS

14-19 September

10th European Conference on Controlled Fusion and Plasma Physics

Moscow, Union of Soviet Socialist Republics

Prot. M.S. Rabinovich, Lebeder Phivsical Institute

21-26 September

12th Europhysics Conference on Macromolecular Physics:

Molecular Mobility in Polymer Systems

Leipzig, German Democratic Republic

Prot W Hoizmuller, Department of Physics, Karl Marx University.

22-25 September

Soft Magnetic Materials (SMM 5)

Grenoble, France

Prof. P. Brissonneau, ENSIEG, Domaine Universitaire
Boite Postale 46. F. 38402 St. Martin dr Hères
EPS Divisions

Quantum Electronics

Atomic Physics

Condensed Matter

Section: Macromolecular

Physics

Astronomy and Astrophysics Solar Section

Nuclear Physics

High Energy and

Particle Physics

Computational Physics

\section{All Divisions and \\ Advisory Committees}

\section{Condensed Matter \\ Section: Semiconductors and Insulators}

Plasma Physics

$$
\begin{gathered}
\text { Condensed Matter } \\
\text { Section: Macromolecular } \\
\text { Physics }
\end{gathered}
$$

\section{Condensed Matter}

Magnetism Section
EPS Divisions, Sections and Group

Astronomy and Astrophysics Division

Solar Section

Atomic Physics Division

Atomic Spectroscopy Section

Chemical Physics

Electronic and Atomic Collisions

Molecular Physics

Computational Physics Group

Condensed Matter Division

Low Temperature Section

Macromolecular Physics

Magnetism

Metals

Semiconductors and Insulators

Surface and Interface

High Energy \& Particle Physics Division

Nuclear Physics Division

Plasma Physics Division

Quantum Electronics Division
Europhysics News is the official journal of the European Physical Society that comprises 28 National Societies, Academies and Groups, over 3000 Individual Ordinary Members and 30 Associate Members. Governing bodies of EPS are the General Meeting, Council and an elected Executive Committee responsible for detailed policy, EPS promotes the collaboration of physicists throughout Europe and encourages all aspects of international exchange in physics. EPS awards scholarships for research and studies and makes arrangements for teaching abroad. EPS publishes, in addition to Europhysics News, Europhysics Conference Abstracts, the Procetdings of its General Conferences and (with the L.O.P ) the European Journal of Physics. Individual Ordinary Members receive Europhysics News (subscription for non-members: $75 \mathrm{Sw}$. Fr./a), substantial rebates on publications and pay reduced fees at conferences. Application for membership is made through the permanent Secretariat in Geneva. Annual subscription for members of a National Society is $32 \mathrm{Sw}$. Fr.
Editor: E.N. Shaw

Meetings Compilation: W.S. Newman

Editorial Board:

K. Appert, A. Baratoff, G.J. Béné,

G.R. Macleod, A. Maeder, J. Muller

Editorial and Advertising Office at the EPS Secretariat.

Address: EUROPEAN PHYSICAL SOCIETY P. O. Box 69 . $\mathrm{CH}-1213$ Petit-Lancy 2

Switzerland

Telephone: Geneva (22) 931130

Telex: $23 \mathbf{4 5 5}$ alarm ch

Cables: europhys genève

Printed by: Pfirter frères SA $\mathrm{CH}-1213$ Petit-Lancy/Switzerland 